$\mathbf{V}_{\mathbf{n}}$

\title{
Fast Aerodynamic Calculations based on a Generalised Unsteady Coupling Algorithm
}

\author{
Daniel Kharlamov *, Andrea Da Ronch ${ }^{\dagger}$, Jernej Drofelnik ${ }^{\ddagger}$, and Scott Walker ${ }^{\S}$ \\ Faculty of Engineering and Physical Sciences \\ University of Southampton, Southampton, SO17 1BJ, U.K.
}

\begin{abstract}
An aerodynamic model for applications to external flows is formulated that provides a great trade-off between computational cost and prediction accuracy. The novelty of the work is the ability to deal with any unsteady flow problem, irrespective of the frequency of motion and motion kinematics. The aerodynamic model, baptised FALCon, combines an in-house unsteady vortex lattice method with an infinite-swept wing Navier-Stokes solver. The two specialised methods are orchestrated by an unsteady coupling algorithm that represents our main research contribution. The paper gives the formulation and algorithmic implementation. FALCon is demonstrated on three test cases of increasing complexity in flow physics, up to flow conditions well outside its validity range. On average, FALCon achieves a computational speed up of a factor of about 50, compared to a full Navier-Stokes run, while capturing relevant flow physics: three-dimensional, viscous, compressible and unsteady phenomena.
\end{abstract}

\section{Nomenclature}

Reduced frequency [-], $k=\omega c_{\text {ref }} / V_{\text {ref }}$

Frequency [Hz]

Aspect ratio of a wing [-], $A R=b^{2} / S$

Taper ratio of a wing [-]

Sweep of the wing $\left[{ }^{\circ}\right]$

Span of the wing $[m]$

Chord length $[\mathrm{m}]$

Freestream velocity [-]

Local freestream angle of attack at $n$-th panel corrected by $\Delta \alpha_{n}[\mathrm{~m} / \mathrm{s}]$

Local VLM panel velocity contribution from unsteady wake in UVLM $[\mathrm{m} / \mathrm{s}]$

\footnotetext{
*PhD student. Currently, Junior Researcher at ISL (French-German Research Institute of Saint-Louis, France). Email: D.Kharlamov@ soton.ac.uk

$\dagger$ Associate Professor, AIAA Senior Member. Email: A.Da-Ronch@soton.ac.uk

$\doteqdot$ Research Fellow. Currently, Aerodynamics Design Engineer at Pipistrel Vertical Solutions, Slovenia.

$\S$ Associate Professor.
} 


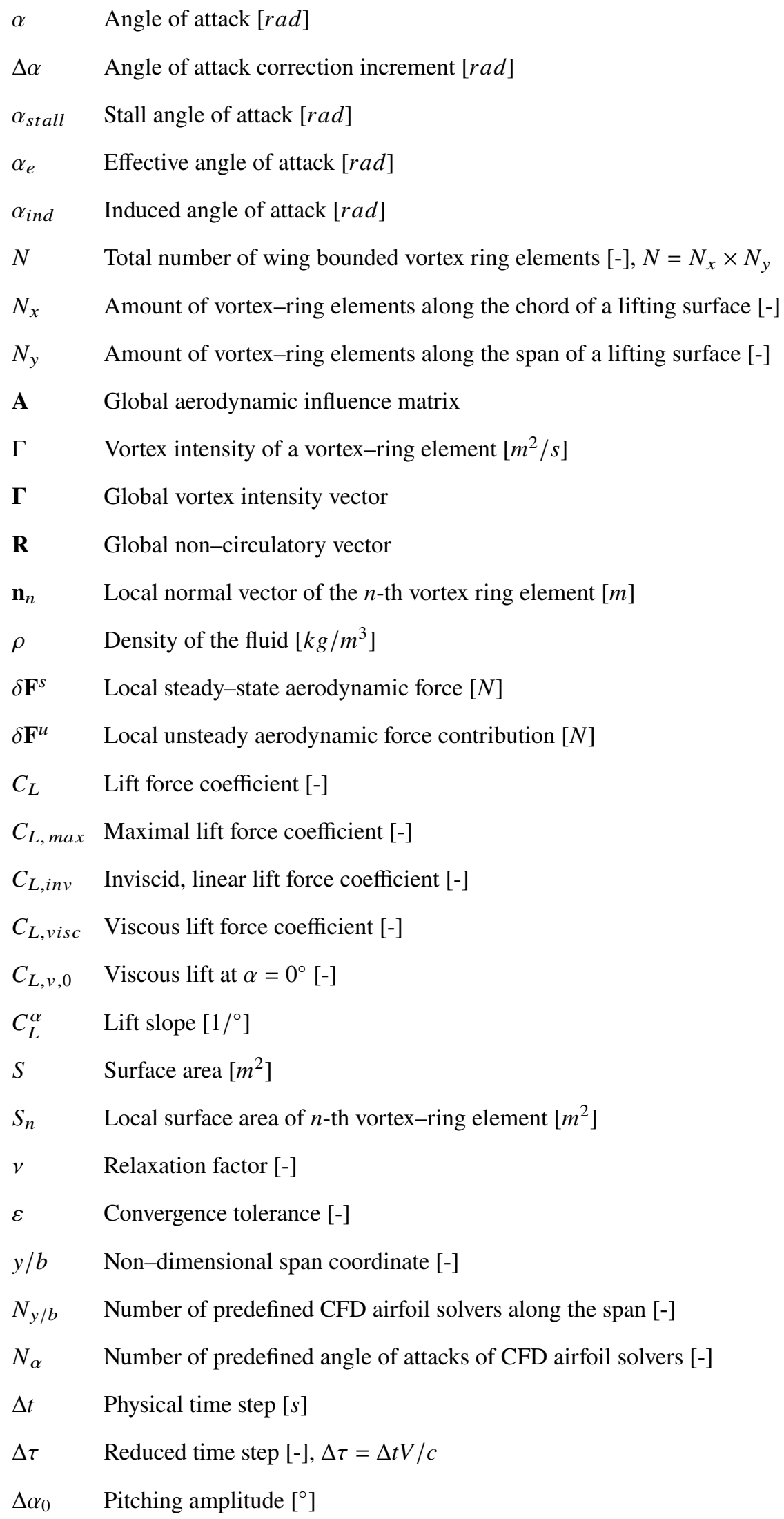




$\begin{array}{ll}\Delta h_{c, 0} & \text { Non-dimensional heaving plunge amplitude [-] } \\ h_{c} & \text { Non-dimensional vertical displacement [-] } \\ M & \text { Mach number [-] } \\ R e & \text { Reynolds number [-] } \\ y^{+} & \text {Dimensionless wall distance [-] }\end{array}$

\section{Introduction}

Aerodynamic models based on linear aerodynamic assumptions have been used in the aerospace industry since the 1960s [1] due to: a) straightforward set-up of the problem, using simplified descriptions of the lifting surfaces; b) low computational effort enabling efficient trade-off studies for envelop searches; and c) its applicability, as the method has been calibrated on a number of existing aircraft configurations to account for unmodelled nonlinear flow phenomena. These models are deployed to support the development of unconventional configurations at the conceptual stage, expediting the exploration and down-selection of a large number of configurations. Higher-fidelity aerodynamic models based on full three-dimensional (3D) Reynolds-averaged Navier-Stokes (RANS) solvers may provide a feasible alternative to existing practice. However, these models are too expensive for routine use to industrial applications owing to the high computational costs involved in solving the RANS equations. Today, they find deployment in the late preliminary to detailed stages, mostly for steady-state problems. A third level of fidelity in aerodynamic analysis tools exists in an attempt to bridge knowledge-based and linear models with higher-fidelity models. A brief historical overview of this mixed-fidelity level follows in the subsequent paragraphs.

The steady-state analysis for a subsonic flow is traditionally performed using the lifting-line model, or models based on linear panel theory. As an extension, the first nonlinear coupling between the lifting-line model and viscous lift curves of airfoils was proposed in 1934 by Tani [2] and in 1938 by Multhopp [3]. In their approach, the circulation intensity of the vortex-ring elements is corrected within the lifting-line model by using sectional viscous data extracted from aerofoil lift curves. This approach ensures a better match of the nonlinear lift slope of a finite wing at higher angles of attack. In 1947, an improved nonlinear lifting-line model, but based on the same approach of [2, 3], was proposed in [4] which iteratively corrects the numerical results obtained by the lifting-line model using nonlinear sectional lift curve data. In 1951, Sivells et al. [5] extended the coupling algorithm to unswept wings including flaps and ailerons into the model. The underlying idea is to correct iteratively the vortex intensity of the wing bounded vortex lattice method (VLM) panels using available two-dimensional (2D), nonlinear aerodynamic data. This method is commonly denoted $\Gamma$-based coupling algorithm. The 3D numerical results of the aerodynamic loads on wings obtained by this approach 
were in good agreement with 3D reference data before the stall angle of attack, $\alpha_{\text {stall }}$. However, the applicability of the $\Gamma$-based coupling algorithm is limited to the maximum wing lift coefficient, $C_{L, \max }$. Beyond stall, the lift curve slope, $C_{L}^{\alpha}$, becomes negative and the solution not unique.

To overcome the non-uniqueness of the $\Gamma$-based coupling algorithm near stall, Tseng and Lan [6] proposed the $\alpha$-based correction method in 1988. Their method couples the lifting-line model or the VLM with sectional aerodynamic data by correcting, at each aerodynamic panel along the wing span, the local freestream angle of attack. The algorithm, however, suffered from poor convergence in post-stall conditions. In the 2000s, Van Dam et al. [7, 8] introduced a revised formulation to improve the convergence properties of the $\Gamma$-based coupling algorithm. More recently, Gallay and Laurendeau [9] [10] extended the formulation to general wing planforms in low- and high-speed of flight, and at high angles of attack.

A formulation for unsteady flows was presented in 2017 [11]. Two approaches, with a precomputed viscous database, were discussed. In cases where the quasi-steady assumption holds valid, the database is computed from RANS calculations. This approach is limited to low reduced frequencies, i.e. heuristically $k<0.1$, and low angles of attack. In the second approach, the database is enriched with information extracted from a set of forced motion calculations at several values of mean angles of attack and frequencies. Limitations of this approach are related to the neglect of flow unsteadiness, which becomes a dominant feature in modern aircraft manoeuvres [12], and to the wing kinematics being restricted to a pure sinusoidal motion. It is also worth noting that time marching the unsteady RANS (URANS) equations for periodic flows, as those developing when a forced motion is imposed, is inefficient [13] given the availability of dedicated frequency-domain methods [14]. The second approach carries a resemblance with the concept of aerodynamic tables used for flight simulation, and the incidental drawbacks preventing the full representation of unsteady flows [15].

The quest for a reliable methodology offering affordable prediction and accurate reconstruction of scalar and vector fields has intensified in the last few years. A reason for this ascending trend is the emergence of open-source, off-the-shelf, machine-learning resources. Beyond studies on applied aerodynamics above-mentioned, fluid mechanics has seen a surge of applications leveraging on data assimilation techniques [16-19]. Common to all approaches, which range from Gradient-enhanced Kriging [19] to deep learning [17] models, is the need to improve the prediction accuracy of a lower fidelity model, which may span the entire spectrum of the fluid equations, by assimilating some, distilled information from a higher fidelity model or available measurements. The enhanced, lower fidelity model is then used to reconstruct the scalar or vector field across the whole spatial domain. In Europe, in particular, data assimilation and machine learning techniques are core to the European Commission funded project "Holistic Optical Metrology for Aero-Elastic Research". . $^{*}$

At the core of our work is the need to develop a general framework for unsteady flow problems. Rather than *HOMER project website: https://cordis.europa.eu/project/id/769237 Grant agreement ID: 769237. 
restricting the admissible kinematics of motion due to underlying simplifications, we have chosen to solve certain implementation challenges to retain generality, in such a way to analyse any arbitrary motion. The paper continues in Section [I] outlining the overall approach and providing implementation details. Then, Section III contains a validation of the specialised aerodynamic models that constitute the computational framework. A demonstration on test cases of increasing complexity is presented in Section IV] Finally, concluding remarks are drawn in Section: $\mathrm{V}$

\section{Formulation}

Analysing the external aerodynamics of a wing configuration to a suitable physical resolution is computationally expensive. To address this challenge, we have developed a methodology that computes rapidly aerodynamic loads for steady and unsteady flows. The methodology is compatible with generally available aerodynamic models, using information and data shared during the aircraft conceptual design, and favouring a seamless integration within an existing environment.

The approach we have followed partitions the complex flow problem into two simpler problems. The first problem provides a quick but global overview of the impact that wing planform design variables have on aerodynamic loads. Design variables common to the aircraft designer are aspect ratio, taper ratio, and sweep/dihedral angles, among others. The second problem solves the relevant flow physics to a desired accuracy around a $2 \mathrm{D}$ wing section. This local representation shall account for viscous and compressible flow effects, as well as thickness effects, otherwise neglected in the general problem. The two separate problems are united by the wing sweep angle, which is a common design parameter to both problems. In our implementation, the global problem is solved using the unsteady VLM (UVLM) that is computationally cheap, described in Section II.A, and the local problem is solved using the infinite-swept wing Navier-Stokes solver, overviewed in Section II.B.

The resulting computational tool, referred to as FALCon, builds on two independent, specialised aerodynamic solvers of different fidelity and computational cost which are brought together by one dedicated coupling algorithm that orchestrates the timely execution of the two solvers in the time domain. The novel coupling algorithm is thoroughly discussed in Section II.C.

\section{A. Vortex Lattice Method}

The VLM follows the classic implementation of Katz and Plotkin [20] and Murray [21]. However, there are certain necessary expedients to transform the classic implementation into one suitable to exchange information with an external, viscous solver. These expedients are highlighted hereafter.

A typical unsteady VLM lattice is shown in Figure 1. The lattice consists of wing bounded and free wake panels which are shed at each time step, $t_{k}=t_{0}+k \Delta t$, into the farfield. The body-fixed frame of reference has the $x$-axis in line with the in-coming flow and the $y$-axis directed towards the wing tip. Each wing bounded panel is identified by the 

panel.

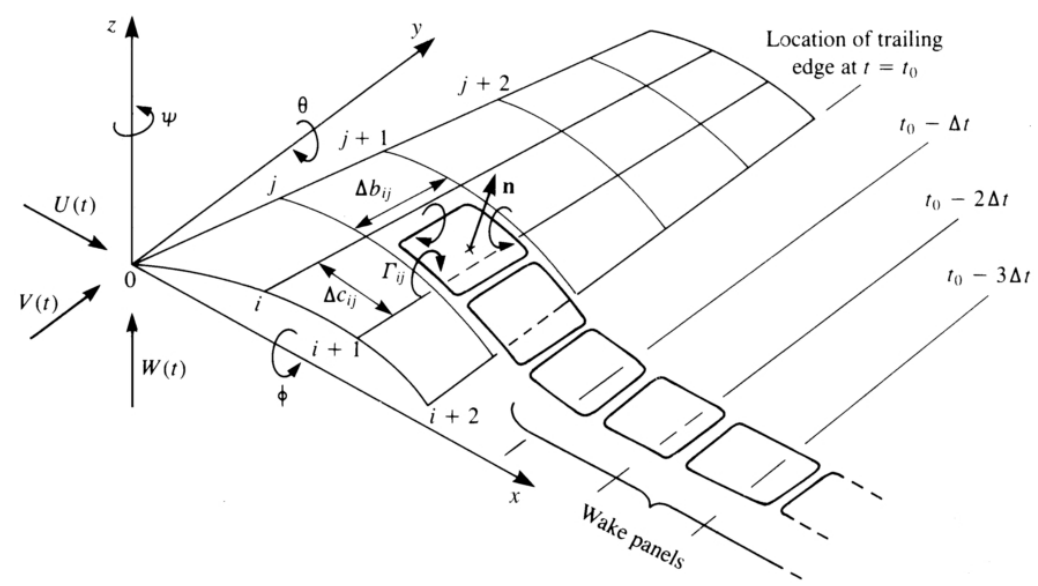

Fig. 1 Unsteady Vortex-Lattice method (Katz and Plotkin [20])

chordwise index, $i$, and the spanwise index, $j$. The vortex ring elements are placed at the quarter-chord line of every

The necessary expedients relate to the calculation of the spanwise distribution of the local angle of attack, $\alpha_{j}$, at the collocation point of a wing bounded panel and the corresponding sectional inviscid lift coefficient, $C_{L, i n v, j}$. These parameters shall be calculated and manipulated independently from the values assigned to freestrem and to neighbouring panels. The local angle of attack at the $j$-th wing bounded vortex panel is computed:

$$
\alpha_{j}=\alpha_{\infty}+\Delta \alpha_{j} \quad j=1,2, \ldots, N_{j}-1
$$

where $N_{j}$ is the number of spanwise wing bounded vortex ring panels. The correction of the local angle of attack, $\Delta \alpha_{j}$, is only relevant when the VLM is coupled with a viscous solver. The parameter $\Delta \alpha_{j}$ interfaces the coupling algorithm and the VLM model.

Using Eq. 11, the local non-circulatory freestream velocity vector at the $j$-th spanwise position of the lattice is:

$$
\mathbf{V}_{\infty, j}=V_{\infty}\left[\cos \left(\alpha_{j}\right), 0, \sin \left(\alpha_{j}\right)\right]^{T}
$$

Here, $V_{\infty}$ is the freestream speed. Further, Biot-Savart law is employed for the calculation of the circulatory velocity contribution, $\mathbf{V}_{n m}$, of the $m$-th vortex ring element on the $n$-th collocation point [21] :

$$
\mathbf{V}_{n m}=\Gamma_{m} \oint \frac{d \mathbf{s}_{m} \times \mathbf{r}_{n m}}{4 \pi\left|\mathbf{r}_{n m}\right|^{3}}=\Gamma_{m} \mathbf{q}_{n m}
$$

The vortex ring intensity is $\Gamma_{m}$ and $\mathbf{s}_{m}$ is the geometrical contour line of the vortex ring of the $m$-th VLM panel. Further, $\mathbf{r}_{n m}$ is the distance vector between the current position on the vortex ring contour of the $m$-th panel and the 
collocation point of the $n$-th panel. The integral is calculated clockwise.

The total local flow velocity at the $n$-th collocation point of a vortex-ring element is:

$$
\mathbf{V}_{n}=\mathbf{V}_{n, a a}+\mathbf{V}_{n, b a}+\mathbf{V}_{\infty, n}+\Delta \dot{\mathbf{x}}_{c, n}
$$

where $\mathbf{V}_{n, a a}$ is the circulatory velocity contribution induced by all wing bounded VLM panels onto the collocation point of the $n$-th wing bounded panel. The term $\mathbf{V}_{n, b a}$ is the circulatory velocity contribution of all wake panels onto the collocation point of the $n$-th wing bounded panel and $\mathbf{V}_{\infty, n}$ is the non-circulatory local freestream velocity contribution (refer to Eq. (2)). Finally, $\Delta \dot{\mathbf{x}}_{c, n}$ is the time derivative of the position of the collocation point in space.

Kinematic boundary conditions are enforced at all collocation points of the wing bounded VLM panels:

$$
\mathbf{V}_{n} \cdot \mathbf{n}_{n}=0
$$

where $\mathbf{n}_{n}$ is the normal vector of a panel placed at the collocation point (see Figure 1 ). This ensures that the potential flow is tangential to the surface of the panel at the collocation point. Combining Eq. (3) with Eq. (4) allows rewriting Eq. (5) for the $n$-th VLM panel as:

$$
\mathbf{n}_{n} \cdot \sum_{m=1}^{N} \Gamma_{m}^{(i)} \mathbf{q}_{n m}=-\mathbf{n}_{n}\left(\mathbf{V}_{\infty, n}\left(\Delta \alpha_{n}\right)+\sum_{m_{w}=1}^{N_{w}} \Gamma_{\text {wake,m }}^{(i-1)} \mathbf{q}_{n m_{w}}+\dot{\mathbf{x}}_{c, n}\right)
$$

Here, $N=N_{i} \cdot N_{j}$ is the total number of wing bounded vortex-ring elements and $N_{w}$ is the total number of all wake panels at a particular time, $t_{i}$. The circulatory wing bounded vortex ring intensity, $\Gamma_{m}^{(i)}$, at the actual time step, $t_{i}$, may be calculated as the vortex ring intensity of the wake panels, $\Gamma_{\text {wake, }}^{(i-1)}$, is known. New panels are shed into the wake during the unsteady process. These panels, departing from the wing trailing edge, have the same vortex intensity as the wing bounded trailing edge vortex elements at a generic time step, $\Gamma_{N_{i+1}, j}^{(i)}=\Gamma_{N_{i}, j}^{(i-1)}$. Then, the new wake panels travel into the farfield with a constant vortex intensity, $\Gamma_{\text {wake,m. }}$. This guarantees the Kutta-Joukowski flow tangency condition at each time step.

Equation (6) is conveniently rewritten in a compact, matrix-vector form:

$$
\mathbf{A} \cdot \boldsymbol{\Gamma}=\mathbf{R}(\Delta \alpha)
$$

where $\mathbf{A}$ represents the aerodynamic influence coefficient matrix and $\mathbf{R}$ is the residual vector of non-circulatory velocity and current wake contributions, updated at each time step. It is worth noting that, for integration to the coupling algorithm, the non-circulatory velocity shall include the increment of the current angle of attack correction, $\Delta \alpha$, from Eq. (6). 


\section{Calculation of Aerodynamic Loads}

The calculation of aerodynamic loads is a prerequisite for the execution of the coupling algorithm. The reason for this is that the algorithm matches, to a user-defined tolerance, the lift coefficient from the inviscid solver, i.e. the VLM, and that from the viscous solver (refer to Eq. (21)).

Aerodynamic loads generated from wing panels are calculated once $\boldsymbol{\Gamma}$ is known. A non-planar formulation based on the Kutta-Joukowski theorem in vectorised form is used [22]:

$$
\delta \mathbf{F}_{n}^{s}=\rho_{\infty} \mathbf{V}_{\infty, n} \times \Delta \boldsymbol{\Gamma}_{n}=\rho_{\infty} \Delta \Gamma_{n} \mathbf{V}_{n} \times \Delta \mathbf{l}_{n} \quad n=1,2,3, \ldots, N
$$

where $\rho_{\infty}$ is the fluid density, $\mathbf{V}_{\infty, n}$ is the local non-circulatory freestream velocity at the collocation point (Eq. 2p and $\Delta \boldsymbol{\Gamma}_{n}$ is the total vortex strength placed at the quarter-chord line of the $n-$ th panel. This total vortex strength can be expressed as $\Delta \boldsymbol{\Gamma}_{n}=\Delta \Gamma_{n} \Delta \mathbf{l}_{n}$, where $\Delta \mathbf{l}_{n}$ is the vector representing the quarter-chord line of the particular VLM panel.

The local vortex strength intensity, $\Delta \Gamma_{i, j}$, of the quarter-chord line of the panel is obtained from the global vector $\boldsymbol{\Gamma}$ as follows:

$$
\Delta \Gamma_{i, j}= \begin{cases}\Gamma_{i, j}-\Gamma_{i-1, j} & \text { if } i>1 \\ \Gamma_{i, j} & \text { if } i=1\end{cases}
$$

For all panels, which are not directly located at the leading edge of the lattice, i.e. $i>1, \Delta \Gamma$ is obtained as difference between the vortex ring intensity of the current panel, $(i, j)$, and the intensity of the corresponding upstream VLM panel, $(i-1, j)$.

In an unsteady analysis, Eq. (8) is augmented with a contribution from unsteady, transient loads [22]:

$$
\delta \mathbf{F}_{n}^{u}=\rho_{\infty} \Delta \dot{\Gamma}_{n} S_{n} \mathbf{n}_{n}
$$

where $\Delta \dot{\Gamma}_{n}$ is the time derivative of $\Delta \Gamma_{n}$ and $S_{n}$ the reference panel area. The time derivative, $\Delta \dot{\Gamma}_{n}$, is approximated with first order, forward Euler method:

$$
\Delta \dot{\Gamma}_{n} \approx \frac{\Delta \Gamma_{n}^{(k)}-\Delta \Gamma_{n}^{(k-1)}}{\Delta t}
$$

As a final step, the lift coefficient at the $j$-th panel is calculated as:

$$
C_{L, i n v, j}=\frac{1}{q_{\infty} S_{j}} \sum_{i=1}^{N_{i}}\left(\delta F_{z, i, j}^{s}+\delta F_{z, i, j}^{u}\right)
$$

where $q_{\infty}=0.5 \rho_{\infty} V_{\infty}^{2}$ is the freestream dynamic pressure, $S_{j}$ is the reference area of the $j$-th panel, and $\delta F_{z, i, j}^{s}$ and $\delta F_{z, i, j}^{u}$ are, respectively, the steady and unsteady lift contributions. 


\section{Calculation of Induced Angle of Attack}

The induced angle of attack, $\alpha_{i}$, plays an important role as part of the coupling algorithm. Practically, the induced angle of attack allows including 3D aerodynamic effects, which are predicted by the VLM, within the sectional viscous data. The calculation of $\alpha_{i}$ is needed at each time step, as represented in Eq. (19).

Following Katz and Plotkin [20], denote $b_{n m}$ the aerodynamic influence coefficient of the $m$-th vortex ring on the collocation point of the $n$-th panel. Then:

$$
b_{n m}=\mathbf{n}_{n} \cdot \tilde{\mathbf{q}}_{n m}
$$

where $\tilde{\mathbf{q}}_{n m}$ is the normalised velocity contribution by the vortex ring intensity, $\Gamma_{m}$, of the $m$-th vortex wing, when only two streamwise vortices of the panel are taken into account. The induced downwash coefficient, $w_{\text {ind }}$, at the collocation point of the $n$-th wing bounded vortex ring element is calculated as:

$$
w_{\text {ind }, n}=b_{n m} \Gamma_{m}
$$

This post-processing step is carried out for all wing bounded vortex-ring elements at each time step. Finally, $\alpha_{i}$ is calculated at the collocation point of every wing bounded vortex-ring panel:

$$
\tan \left(\alpha_{i n d, n}\right)=-\frac{w_{i n d, n}}{V_{\infty}}
$$

As a final consideration, a careful implementation of the above-mentioned expedients ensures accessing the spanwise distribution of the induced angle of attack, $\alpha_{i n d, j}$, and that of the inviscid lift coefficient, $C_{L, i n v, j}$, at each VLM panel. These quantities will be feed in to the coupling algorithm.

\section{B. Infinite-swept Wing Navier-Stokes Solver}

In the interest of conciseness, a high-level presentation of the infinite-swept wing Navier-Stokes solver is here given. The reader shall consult Ref. [23] for further details here irrelevant.

In a 2D analysis, the flow solution does not depend on the third direction. Practically, this approach is restrictive when the wing cross-section is swept from the in-flight direction (or the direction of the in-coming flow). Cross-flow effects become dominant, and greatly affect the flow development around the wing cross-section. The infinite-swept wing Navier-Stokes solver is developed for these cases in mind. It relaxes the assumptions of a 2D solver, only enforcing the flow to be uniform along the third direction. The flow component along the third direction is calculated as part of the solution process, rather than being prescribed as in a $2 \mathrm{D}$ analysis.

Advantages of the infinite-swept wing Navier-Stokes solver are two-fold. The solver allows investigating a greater 
generality of flow conditions around unswept and swept wings, employing the same 2D grid traditionally used in a classical, 2D analysis. The implementation of this specialised solver is easily done starting from a 2D or 3D flow solver. Geometric sweep effects are included through a thickening of the wing cross-section.

All results here reported were generated using the DLR-tau flow solver. Our group at Southampton implemented and integrated the infinite-swept wing Navier-Stokes solver into DLR-tau. Due to its computational efficiency compared to existing state-of-the-art options, the implementation is denoted as $2.5 \mathrm{D}+$. The steady and unsteady $2.5 \mathrm{D}+$ flow solver was demonstrated [23] around single and multi-element wing sections in low- and high-speed of flight, using laminar and various turbulence models. The flow solver was applied to study transonic buffet for a family of wing configurations [24] and in aerodynamic shape optimisation using solvers of different fidelity [25]. Since its deployment within an industrial environment, the 2.5D+ flow solver has been used in excess of hundreds of thousands of times for production.

\section{Unsteady Coupling Algorithm}

Having described two specialised aerodynamic models of different fidelity, we now turn our attention to the coupling algorithm that represents the novelty of this paper. The formulation of the coupling algorithm builds upon the steady-state, $\alpha$-based coupling algorithm. A schematic of the unsteady coupling algorithm is shown in Figure 2. Confronted with the steady-state version, the unsteady counterpart features an outer time loop to advance the two aerodynamic models in time. The algorithm, designed to spawn new processes, connect to their input/output/error pipes, and obtain their results, is structured in two main parts. These are discussed thoroughly in the following sections. At the core of the unsteady coupling algorithm is the ability to deal with any arbitrary, a-priori unknown motion in order to overcome limitations of current state-of-the-art alternatives.

\section{Part I: Synchronous Management of Aerodynamic Models}

The overarching goal of Part I is to manage data generation from two specialised and separate aerodynamic models. The different aspects of coordinating the time integration of the models are seen as elements of an interdependent process, managing them in a manner to optimise the performance of data generation for any arbitrary motion.

Part I supervises critical aspects, such as, advancing the UVLM and URANS solutions in the time domain, adopting the same time step size. For cases with geometry motion, the position and rotation of the planar VLM lattice is trivially updated. Moving the 2D grid of the URANS solver is done by a rigid translation and rotation. This method is preferred over alternatives because, in all cases, it avoids warping the mesh or re-meshing the grid, operations that would otherwise become necessary and add computational cost when performing an aeroelastic analysis with a 3D grid. It is worth noting that a translation, i.e. in-plane or out-of-plane bending, and a rotation, i.e. torsion, are fundamental components to reconstruct any general type of motion. Motion rates have an impact on flow unsteadiness and shall be 


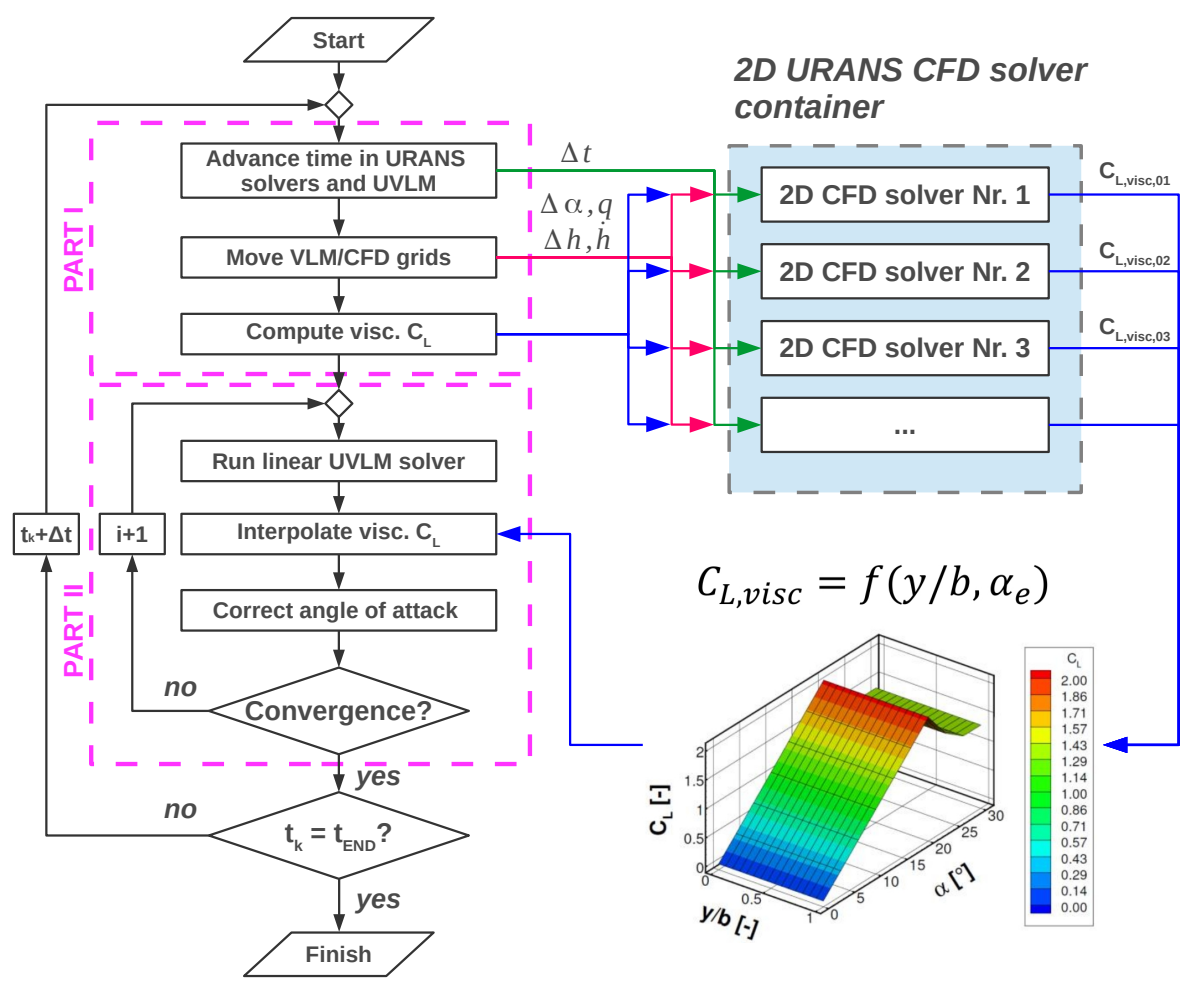

Fig. 2 Schematic of the unsteady coupling algorithm: Part I performs synchronous management of aerodynamic models, and Part II executes data fusion 
included. In the UVLM, the velocity of the collocation point of every wing bounded panel, $\Delta \dot{\mathbf{x}}_{c, n}$, contributes to Eq. (4) and is calculated with first order, forward Euler method:

$$
\dot{\mathbf{x}}_{c, n} \approx \frac{\mathbf{x}_{c, n}^{(k)}-\mathbf{x}_{c, n}^{(k-1)}}{\Delta t}
$$

In the URANS solver, motion rates are imposed through additional unsteady whirl-flux velocities calculated once the motion at the current time step is known. As a result, FALCon offers the capability to analyse any arbitrary motion, whether prescribed or unknown a-priori.

Managing data generation is the most challenging aspect of the coupling algorithm. The view is to have a database, or container, of unsteady, viscous aerodynamic data which is interrogated on-demand during the iterative process coordinated by Part II (further details follow in Section III.C.2). Confronted with existing alternatives already discussed in the Introduction, our implementation follows the most general approach which comes with a number of implementation challenges. With support of Figure 3 consider a wing with a cross section that varies along the span. As an example, three sections (20,50 and $80 \%$ of the span) are considered where sectional aerodynamic characteristics are sought after. At the core of Part I is the ability to compute the dependence of the unsteady, sectional lift coefficient on the instantaneous angle of attack and on relevant motion rates, at each particular wing section. The dependence on the angle of attack is not unexpected because our approach builds upon the steady-state, $\alpha$-based coupling algorithm. From Figure 3 Part I spawns 15 URANS analyses, grouped into three spanwise sections. Initially, the viscous database stores data from 15 RANS analyses which are initialised from different freestream values of the angle of attack. Figure 3 shows an exemplary range chosen between -8 and $8^{\circ}$. The initial database is steady-state and does not include unsteady effects. To overcome this limitation, at each time step iteration, $t_{k}$, the instantaneous motion and rates become known. Then, Part I spoons this information to all URANS analyses (which are marched in time using dual-time stepping [26]) and a new database valid at $t_{k}$ is generated. Data are stored in a tabular form that visually takes the form of a carpet plot (see insert in Figure 2) representing the sectional, viscous $C_{L}\left(t_{k}\right)$ versus $\alpha\left(t_{k}\right)$ and $y / b$. The dependence on motion rates is omitted for brevity. As explained in the next Section, Part II will access this database to extract, at each wing section $y / b$, the viscous lift coefficient at the current time step, $t_{k}$, interpolated at a specified value of the effective angle of attack:

$$
C_{L, v i s c}\left(t_{k}\right)=f\left(\alpha_{e}\left(t_{k}\right), y / b\right)
$$

Particular attention shall be devoted to two considerations. The first is for the initial angle of attack range, see Figure 3. Part II accesses the viscous aerodynamic database with a value of $\alpha_{e}\left(t_{k}\right)$ calculated from Eq. [19]. The iterative nature of data fusion, exemplified by Eq. 21, , proceeds through variations or corrections of the angle of attack. It is therefore critical to have a sufficiently wide range to avoid hampering the convergence of the algorithm (i.e. 
interpolation is preferred over extrapolation). The second consideration relates to the motion. There are cases where the motion is unknown a-priori, such as in flight dynamics or aeroelasticity. An external, dedicated model will then be responsible to calculate the motion and rates for a set of aerodynamic loads calculated at $t_{k}$. The motion information is then spooned to Part I that continues the above-mentioned process.

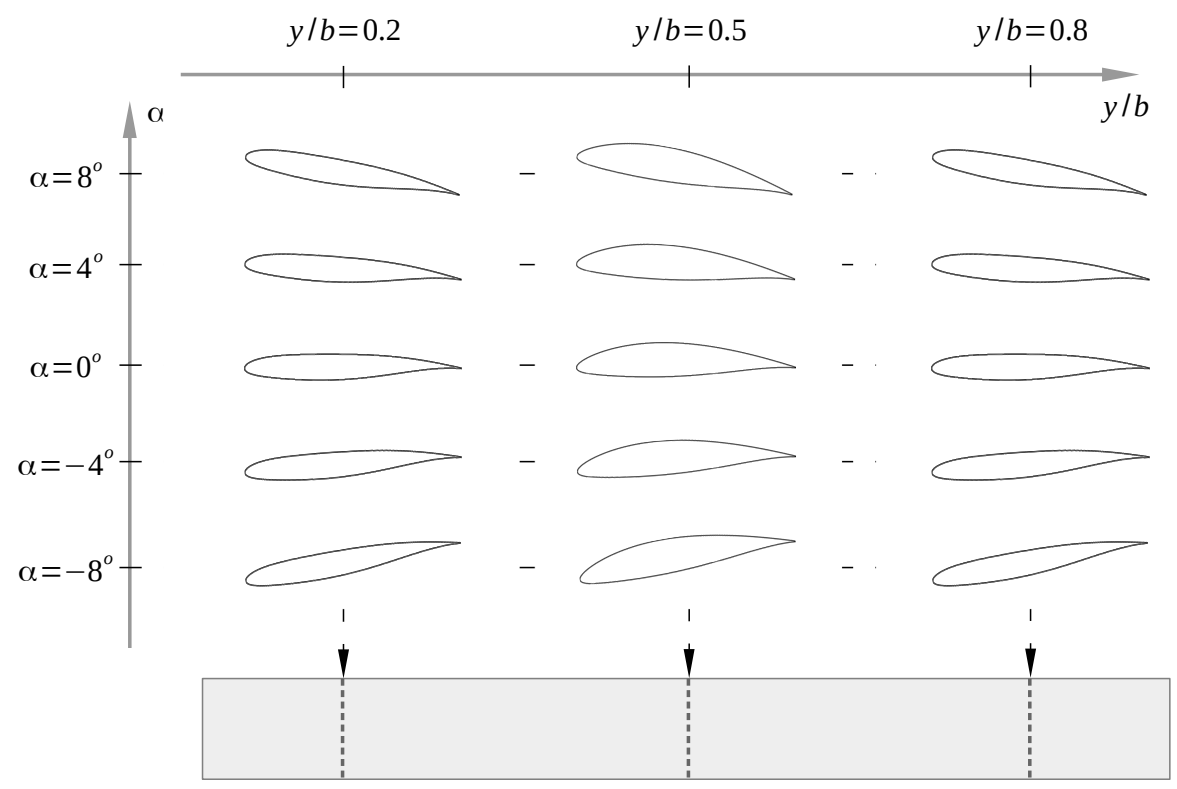

Fig. 3 Schematic of the tabular format of the viscous aerodynamic container, showing dependence on angle of attack and spanwise location. Dependence on motion rates is not represented for simplicity

\section{Part II: Data Fusion}

At the core of Part II is the process of integrating aerodynamic data from two aerodynamic sources to produce more consistent and accurate information than that provided by any individual data source. For steady-state problems, one turns to Van Dam's $\alpha$-based coupling algorithm [7]. The literature is scarce when unsteady problems are considered.

A possible approach is to resort to the calculation of the sectional, effective angle of attack, $\alpha_{e, j}$, using the formulation [27]:

$$
\alpha_{e, j}=\frac{C_{n}}{2 \pi}
$$

where $C_{n}$ is the unsteady, sectional normal force coefficient from the inviscid solver. The caveat of this simple relationship is that $C_{n}$ shall relate to the circulatory contribution only, but the UVLM does not separate the circulatory contribution from the non-circulatory one [28]. As a result, we have observed an overestimation of $\alpha_{e, j}$ which leads to a significant overprediction of the unsteady, sectional, viscous lift coefficient, $C_{L, v i s c}$. Our suggestion is to consider the 
alternative:

$$
\alpha_{e, j}=\alpha_{\infty}-\left.\alpha_{i n d, j}\right|_{(c / 4)}
$$

where $\alpha_{\infty}$ is the nominal freestream angle of attack. The second term on the right hand side is the induced angle of attack at the quarter chord of the wing section calculated using the inviscid solver. Generally, the VLM formulation calculates the induced angle of attack at the collocation point of wing bounded panels. To obviate this conflict, the interpolated value at the wing sectional quarter chord is based on interpolation of the values at the collocation points of two or more chordwise panels, $N_{i}>1$.

At the $j$-th wing section, the instantaneous, viscous lift coefficient $C_{L, v i s c}$ is extracted from the viscous database created at the actual time step, $t_{k}$. The database is interrogated with the value of $\alpha_{e, j}$ obtained from Eq. (19). Then, the angle of attack correction at the $j$-th wing section is estimated for the next time step, $t_{k+1}$ :

$$
\Delta \alpha_{j}^{(k+1)}=\Delta \alpha_{j}^{(k)}+v \frac{C_{L, v i s c}^{(k)}-C_{L, i n v}^{(k)}}{2 \pi}
$$

where $v$ is the relaxation factor used to control the update of the variable at each inner iteration. As depicted in Figure 2 . Part II supervises the convergence behaviour of the pseudo-iterations. Convergence at each time step is achieved when the lift coefficient from the two aerodynamic sources, at all spanwise wing stations, meets the following condition

$$
\left\|C_{L, v i s c, j}-C_{L, i n v, j}\right\|<\varepsilon
$$

The parameter $\varepsilon$ is a user-defined tolerance regulating solution accuracy.

\section{Validation}

This section concerns the validation of the UVLM for a set of canonical problems: a suddenly accelerated flat plate of different aspect ratios, and a thin aerofoil undergoing forced motion in two degrees of freedom. The validation of the infinite-swept wing Navier-Stokes solver is here omitted for brevity. The reader eager to learn more details about this is encouraged to examine Ref. [23].

\section{A. Suddenly accelerated flat plate}

The lift coefficient build-up for a suddenly accelerated flat plate is reported in Figure 4 for values of the aspect ratio $(A R)$ from 4 to infinity. The VLM lattice consists of 4 chordwise panels and 26 spanwise panels, equally distributed between root and tip. When $A R=\infty$, the flow is fully 2D. Practically, the UVLM uses a sufficiently large aspect ratio $(A R=100)$ combined with one spanwise panel to neglect modelling $3 \mathrm{D}$ effects. A nondimensional time step $\Delta \tau=1 / 16$ is used for all unsteady analyses, and the freestream angle of attack is $5^{\circ}$. The initial impulsive part and the 


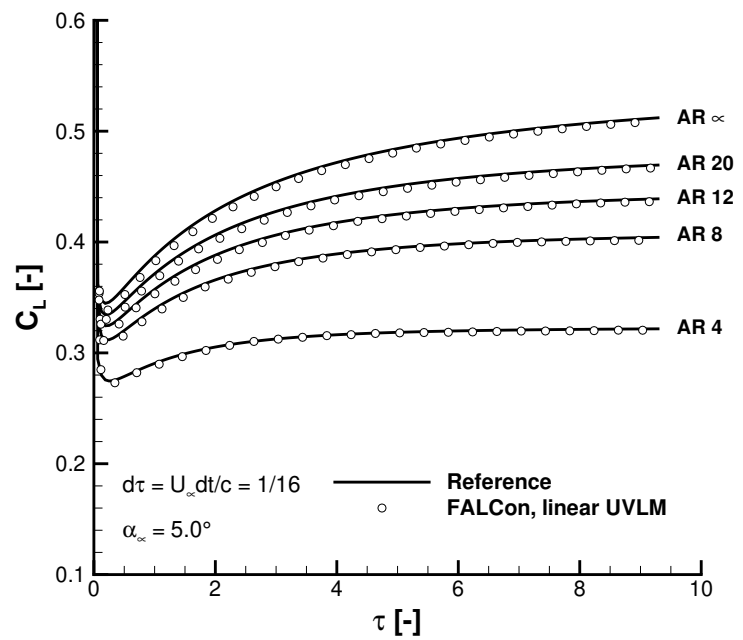

Fig. 4 UVLM validation: suddenly accelerated flat plate. Reference data from [20]

\section{B. Forced motion of a thin aerofoil in pitch and plunge}

The second validation test case is for a thin aerofoil undergoing a prescribed motion. The test case introduces the dependence on two degrees of freedom that are independently excited and on the reduced frequency of oscillations, $k=\omega c / V_{\infty}$.

For an harmonic motion, the time history of the pitch degree of freedom is defined by:

$$
\alpha(\tau)=\alpha_{0}+\alpha_{A} \sin (2 k \tau)
$$

where $\alpha_{0}$ is the mean value and $\alpha_{A}$ the amplitude of oscillations. Similarly, for plunge:

$$
\xi(\tau)=\xi_{0}+\xi_{A} \sin (2, k \tau)
$$

where $\xi=h / c$ represents the nondimensional plunge or heave. Two types of motion are here considered: 1) a pure pitch around the quarter-chord point, defined by parameters $\alpha_{0}=0^{\circ}$ and $\alpha_{A}=2.5^{\circ}$; and 2) a pure plunge, $\xi_{0}=0$ and $\xi_{A}=0.1$. For both types of motion, three values of the reduced frequency are used: $k=0.5,1.0$ and 1.5 . In the UVLM, the 2D aerodynamics around the thin aerofoil is obtained using one panel in the spanwise direction, as in the previous Section for $A R=\infty$. The number of chordwise panels was determined by a convergence study, similarly to Ref. [29], summarised in Table 1] It is worth noting that the nondimensional time step satisfies the relation $\Delta \tau=1 / N_{i}$. This ensures that the chord length of new panels shed into the wake is equal to the chord length of wing bounded panels. 


\begin{tabular}{cccc}
\hline \hline$k$ & $N_{i}$ & $N_{j}$ & $\Delta \tau[]$ \\
\hline 0.5 & 25 & 1 & 0.0400 \\
1.0 & 28 & 1 & 0.0357 \\
1.5 & 42 & 1 & 0.0238 \\
\hline \hline
\end{tabular}

Table 1 UVLM validation: space and time discretisation parameters

Hysteresis loops are shown in Figure 5. Initial transients were removed to highlight the periodic nature of the lift coefficient. Reference data are obtained using Theodorsen's analytical model [30]. One observes a good match between the analytical curves and the UVLM for all cases considered, ranging from low to high values of the reduced frequency.

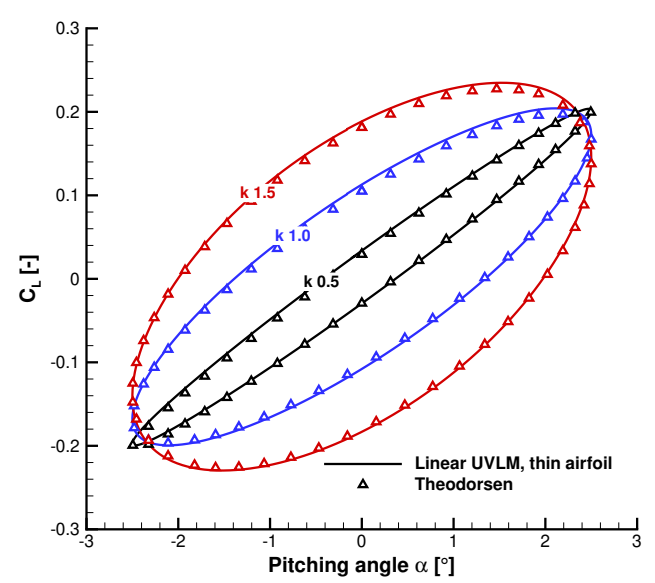

(a) Pitch motion

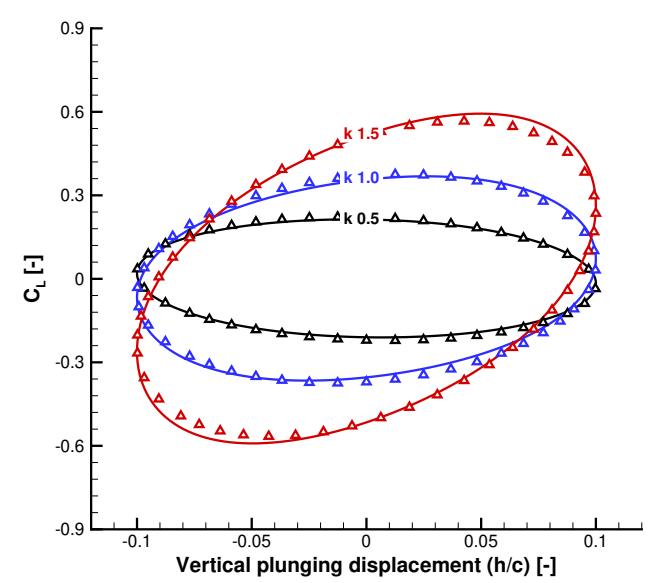

(b) Plunge motion

Fig. 5 UVLM validation: forced motion of a thin airfoil. Reference data reproduced from [30]

\section{Results}

This Section demonstrates the applicability of the complete computational tool FALCon to a number of test cases of increasing complexity. The physics ranges from incompressible to compressible flows in a 2D and 3D setting, driven by unsteady motions at different reduced frequencies. Reference data are obtained from DLR-Tau code solving the URANS equations. The turbulence model is based on Spalart-Allmaras model [31].

\section{A. Forced harmonic motion in pitch and plunge: infinite-span wing}

The first demonstration test case is for a pitching and plunging NACA 2412 aerofoil (infinite-span wing). The compressible flow, which features a weak shock wave, is for $M=0.7$ and $R e=5.5 \cdot 10^{6}$. The harmonic motions are prescribed at low and high values of the reduced frequency, respectively, $k=0.025$ and 0.750 . In pitch, $\alpha_{0}=0^{\circ}$ and $\alpha_{A}=2.51^{\circ}$, and in plunge, $\xi_{0}=0$ and $\xi_{A}=0.1$. The pitch axis is at the quarter-chord point.

Reference data are obtained from time marching the 2D URANS equations. The O-type, structured grid in Figure $6 a$ 
used in all calculations was chosen to guarantee grid independent results. Three levels of grids were generated, from about 25 thousand to 70 thousand grid points. The coarser grid was found adequate to model the unsteady lift coefficient, as reported in Figure 6b. The convective RANS flux is discretised using the second-order central scheme. For time integration, the dual time-stepping scheme is used. The $2 \mathrm{w}-$ cycle multigrid scheme with 50 quasi-steady subiterations per physical time step is chosen at a Courant-Friedrichs-Lewy number of 2.5.

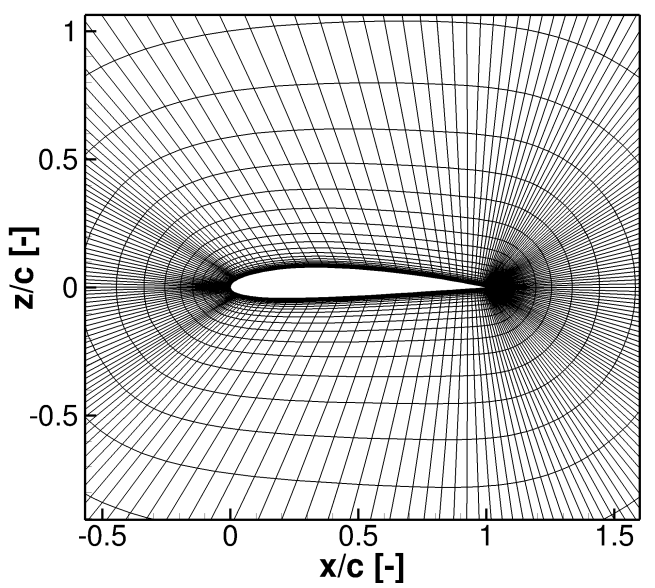

(a)

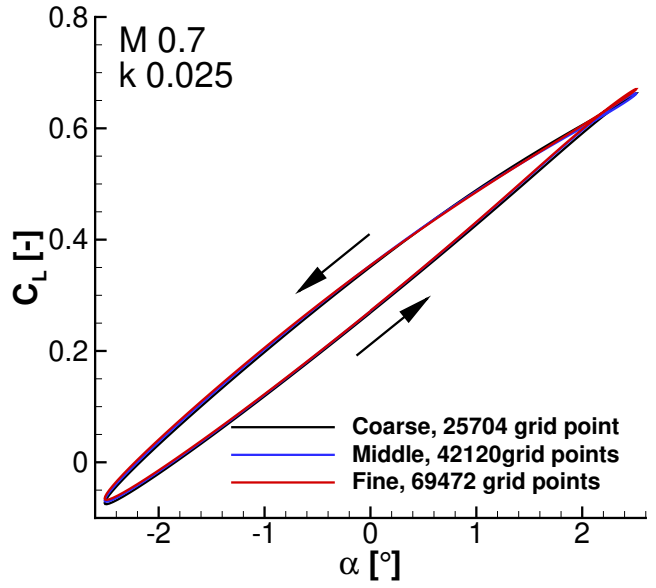

(b)

Fig. 6 Test case A: in (a), coarse 2D grid used in all calculations; and in (b), grid convergence study ( $M=0.7$ and $k=0.025$ )

The analysis setup for FALCon is as follows. The UVLM lattice consists of one single panel $\left(N_{j}=1\right.$ and $\left.N_{i}=1\right)$ of high aspect ratio $(A R=100)$ motivated by the $2 \mathrm{D}$ aerodynamics of this test case. For the viscous solver, numerical parameters are identical to those above-mentioned for the reference data. For this 2D case, the coupling algorithm initially spawns one URANS analysis at the static freestream angle of attack of $\alpha_{0}=0^{\circ}$. As for the 2D nature of the flow, Eq. (19) of the unsteady, $\alpha$-based coupling algorithm simplifies to:

$$
\alpha_{e, j}=\alpha_{\infty}=\alpha_{0}
$$

after eliminating the induced angle of attack from the original formulation. For the coupling algorithm, the relaxation factor is set to $v=0.1$, and the convergence criterion at each time step is defined by the parameter $\varepsilon=10^{-5}$.

Figure 7 reports the lift coefficient hysteresis loops. Initial transients were removed and arrows indicate the direction of the loops. One finds an excellent agreement between FALCon results and reference data, for all reduced frequencies and motions. This indicates a correct conception and implementation of the unsteady coupling algorithm. 


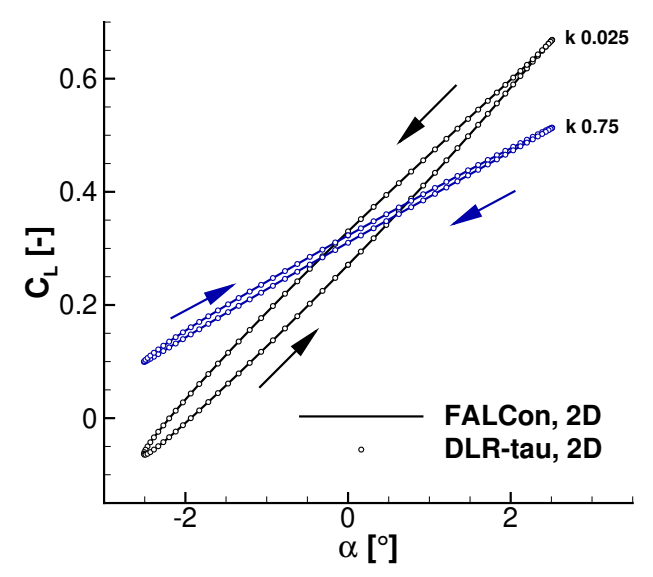

(a) Pitch motion

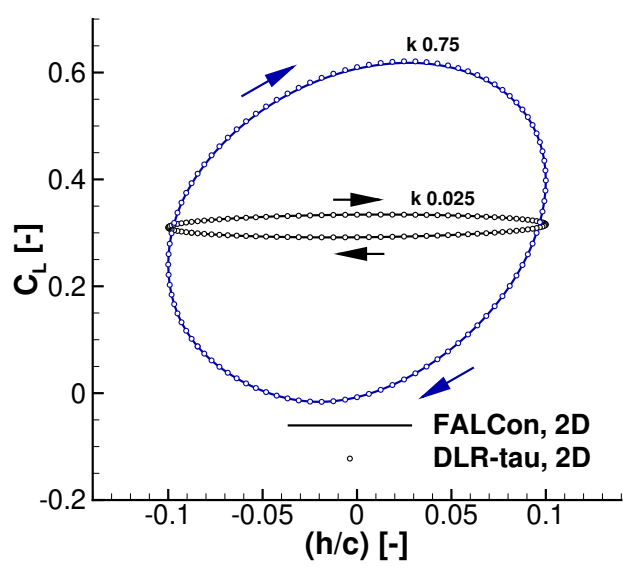

(b) Plunge motion

Fig. 7 Test case A: lift coefficient hysteresis loops for forced harmonic motion at $M=0.7$. In the legend, "DLR-tau, 2D" denotes reference data

\section{B. Forced harmonic motion in pitch and plunge: finite-span wing}

The second test case is for a finite-span, unswept and untapered wing. The aspect ratio is 10 . The wing cross-section is the NACA 2412 aerofoil. The forced harmonic motion in pitch and plunge has the same parameters described in Section IV.A. The pitch axis is at the wing quarter-chord. The test case is run at three values of Mach number $(M=0.3$, 0.5 and 0.7$)$ keeping the Reynolds number fixed at $5.5 \cdot 10^{6}$. This allows investigating the effect of compressibility and the impact that mild shock waves appearing and disappearing during the forced motion have on the predictive performance of the hybrid aerodynamic solver.

Reference data were obtained solving the 3D URANS equations. Results were calculated for three cycles, using 100 time steps per cycle, to achieve a periodic flow state after the decay of initial transients. The maximum number of pseudo-iterations per time step was set to 600 , and the single grid method was used. Other relevant numerical settings are the same as those described in Section IV.A. Figure 8 shows the 3D grid. The grid point distribution at the symmetry plane reuses the coarse 2D grid from Figure 6a. This grid was then extruded in the spanwise direction. It was found that a discretisation with 63 layers of 2D planes, along the wing span, was adequate for grid independent results, as documented in Figure 10a The final 3D grid consists of about two million grid points.

For the hybrid aerodynamic solver FALCon, the VLM lattice consists of $N_{i}=2$ and $N_{j}=100$ panels, refer to Figure 9. Coarser grids revealed a tendency to overestimate the lift coefficient at the lowest and highest values of the angle of attack, as shown in Figure $10 \mathrm{~b}$. The coupling algorithm spawns nine URANS analyses uniformly distributed between -6 and 2 degrees angle of attack. The angle of attack range considers the asymmetric nature of the aerofoil and the expected variation of the effective angle of attack which decreases moving from the symmetry plane to the wing tip. The relaxation factor is $v=5 \cdot 10^{-2}$, and the convergence criterion at each time step is based on the tolerance $\varepsilon=10^{-6}$.

We now focus on the reproducibility of the flow physics using the hybrid aerodynamic solver. Reference data 


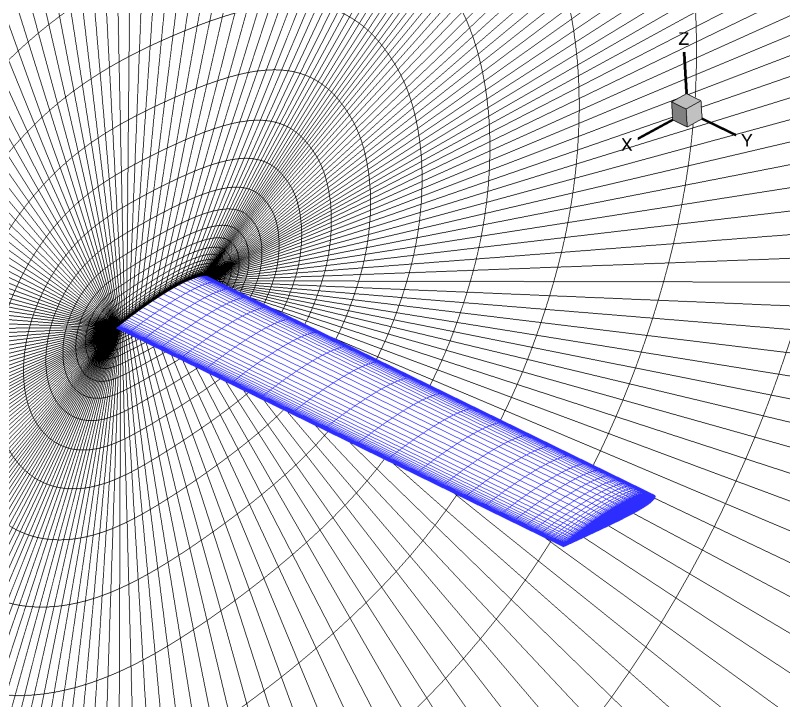

(a) Symmetry plane and wing surface

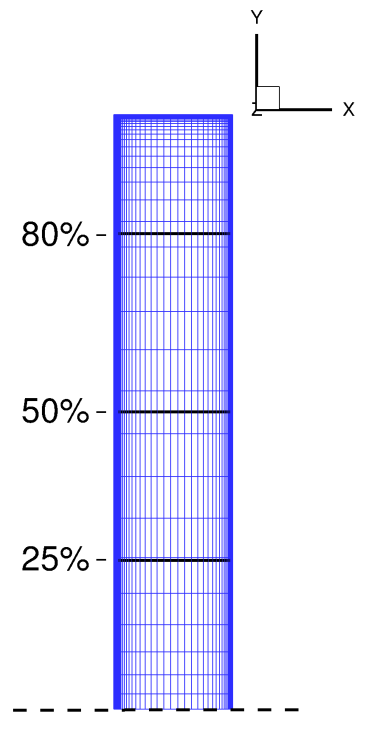

(b) Top-down view

Fig. 8 Test case B: views of the 3D structured grid; in (b), the 3 sections at 25, 50 and 80\% of the wing span are also highlighted

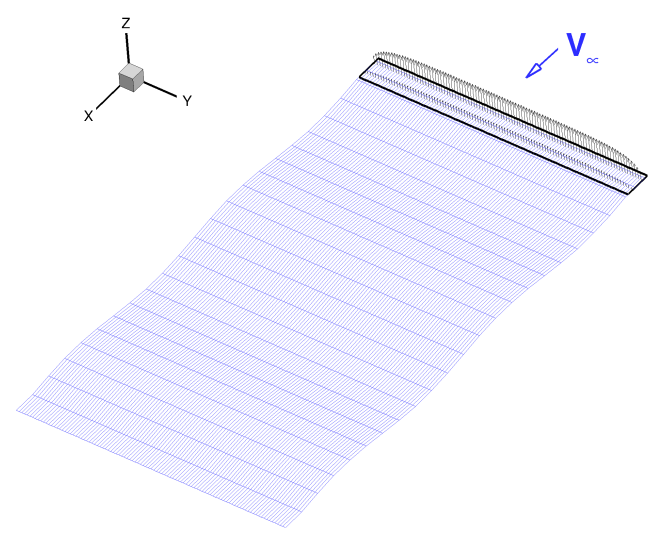

Fig. 9 Test case B: distribution of VLM panels on the wing and in the wave 


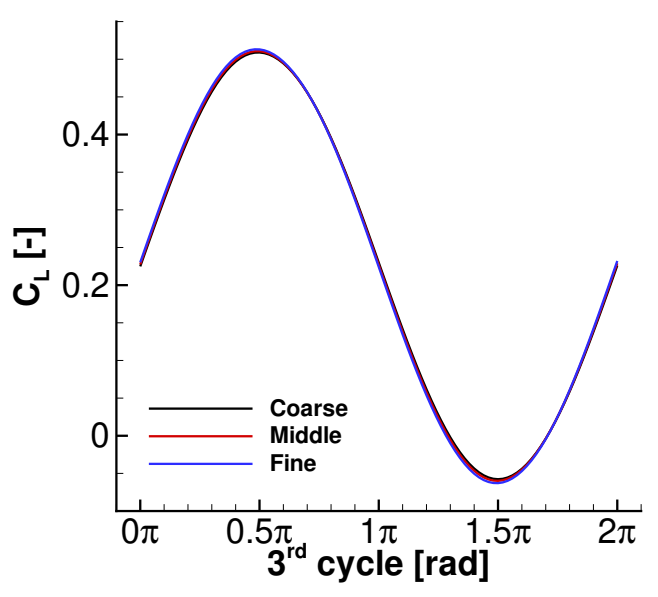

(a) 3D structured grid

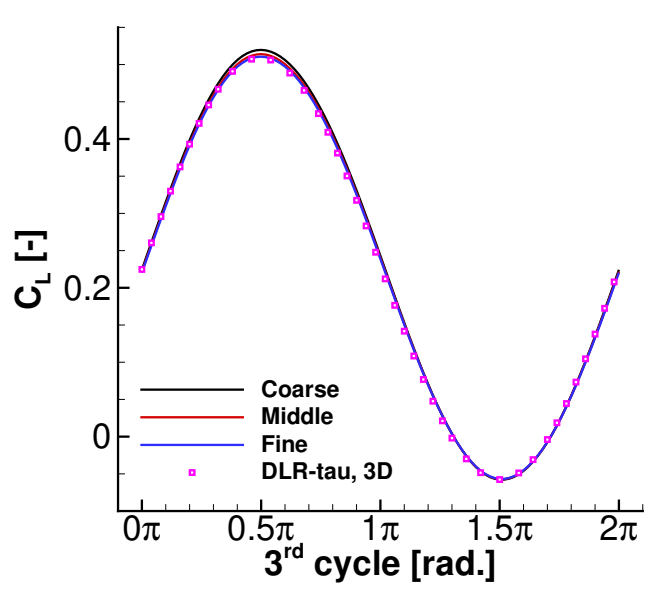

(b) VLM lattice

Fig. 10 Test case B: grid convergence results for pitch motion $(M=0.7$ and $k=0.025)$. In (a), the coarse 3D grid contains about 2 million points, the medium grid 4.2 million points and the fine grid 9 million points; in (b), the coarse VLM lattice consists of $2 \times 25$ panels, the medium lattice $2 \times 50$ panels and the fine lattice $2 \times 100$ panels

are from time marching the 3D URANS equations. Figure 11 reports time domain results for the forced motion in pitch. Qualitatively, the fundamental characteristics associated with flow unsteadiness, i.e. reduced frequency, and compressibility, i.e. Mach number, are well captured in FALCon. In particular, the hybrid aerodynamic solver correctly captures the following elements: 1) the increase of the hysteresis loops for increasing reduced frequency, and the decrease of this trend for increasing Mach number; 2) although flow unsteadiness is marginal at the lowest reduced frequency, $k=0.025$, aerodynamic damping [13] switches sign between $M=0.5$ and $0.7 ; 3$ ) for increasing reduced frequency, loops tilt anticlockwise and the aerodynamic stiffness, related to the loop mean slope, increases. Reproducing these features, in particular point 2 above, guarantees the suitability of the hybrid aerodynamic solver for future aeroelastic analysis. In Figure 12, results for the forced motion in plunge are compared. Qualitatively, the impact of reduced frequency and Mach number on aerodynamic loops are reproduced. Quantitatively, we define the percent reconstruction error $\epsilon$ as:

$$
\epsilon=\frac{1}{N} \sum_{k=1}^{N} \frac{\left\|C_{L, k}^{\mathrm{URANS}}-C_{L, k}^{\mathrm{FALCon}}\right\|}{\bar{C}_{L}^{\mathrm{URANS}}} \cdot 100
$$

where the percent reconstruction error over one cycle is normalised by the time averaged value of the lift coefficient from the 3D URANS analysis, $\bar{C}_{L}^{\mathrm{URANS}}$. The largest values of $\epsilon$ are found at the highest Mach number, and these are summarised in Table 2. For all cases tested, $\epsilon$ is well below 10\%, apart from the single occurrence at $M=0.7$ and $k=0.750$ for the plunging motion.

The hybrid aerodynamic solver offers the appealing feature to reconstruct distributed flow features at any spanwise location which does not coincide with the sections (refer to Figure 8) used to generate the viscous database. This is done at no extra cost because the existing database, at any generic time instant, is simply interrogated with the effective angle 


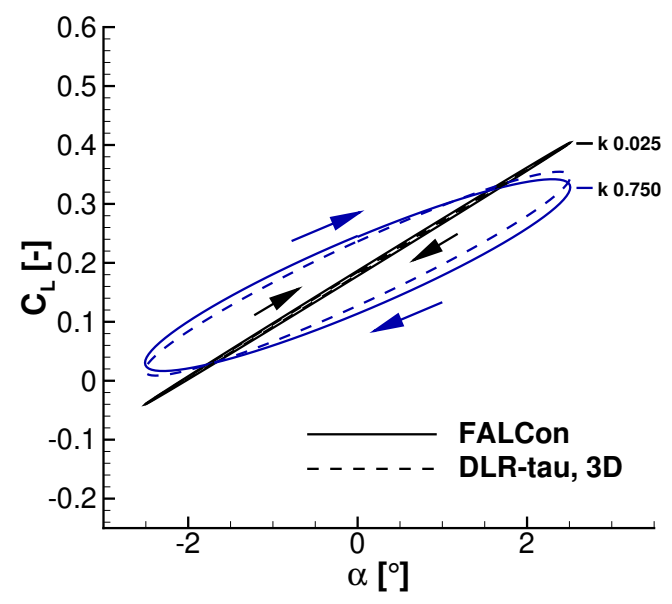

(a) $M=0.3$

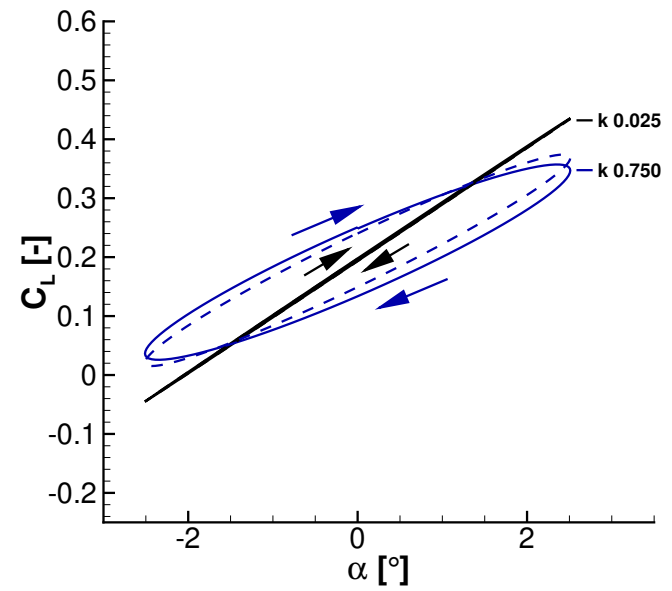

(c) $M=0.5$

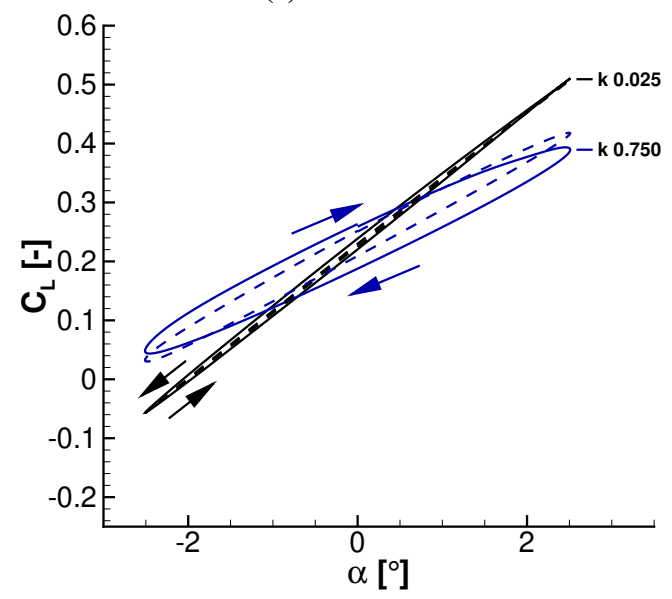

(e) $M=0.7$

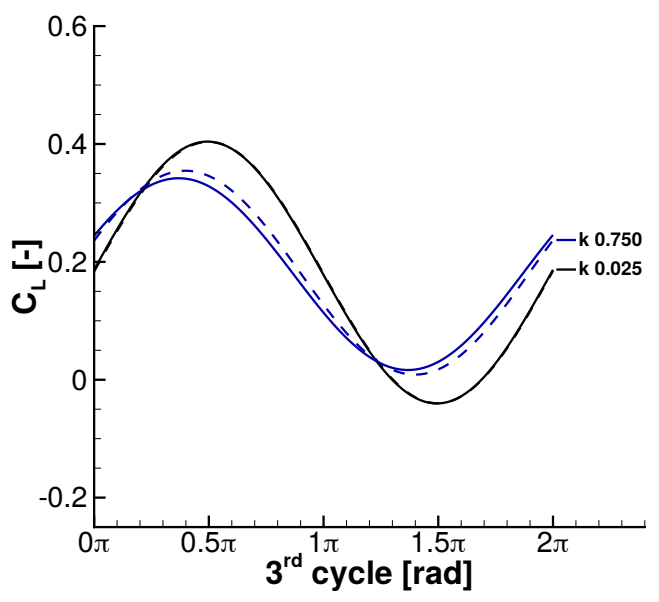

(b) $M=0.3$

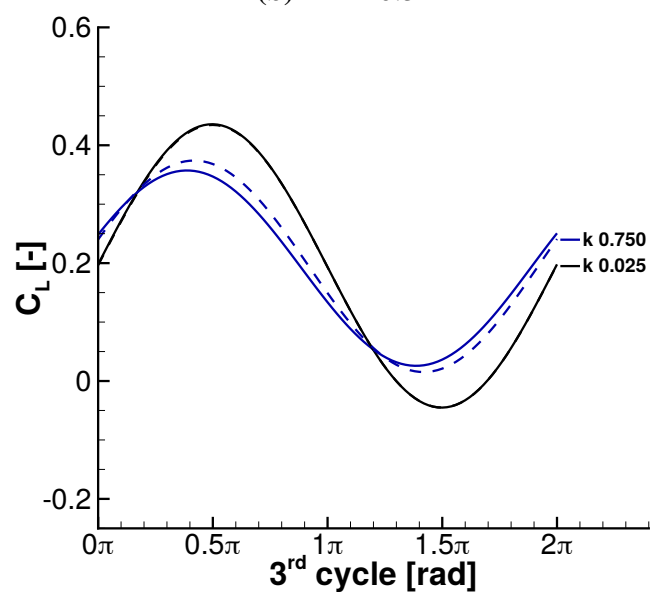

(d) $M=0.5$

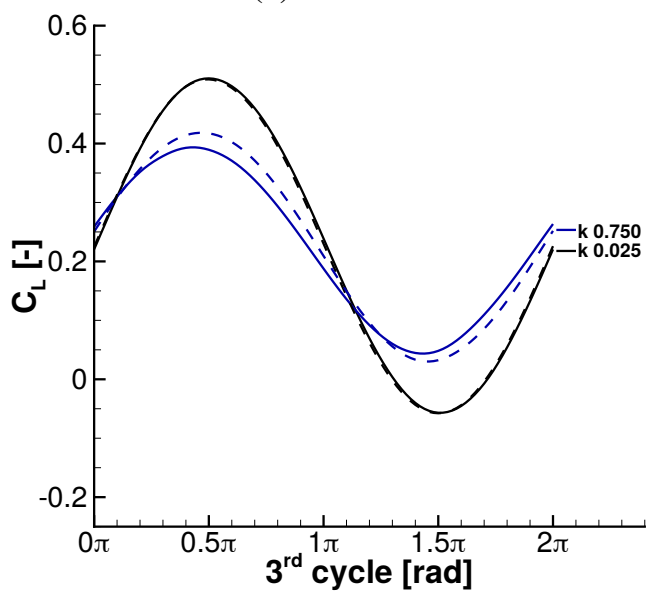

(f) $M=0.7$

Fig. 11 Test case B: lift coefficient hysteresis loops for forced pitch motion. In the legend, "DLR-tau, 3D" denotes reference data 


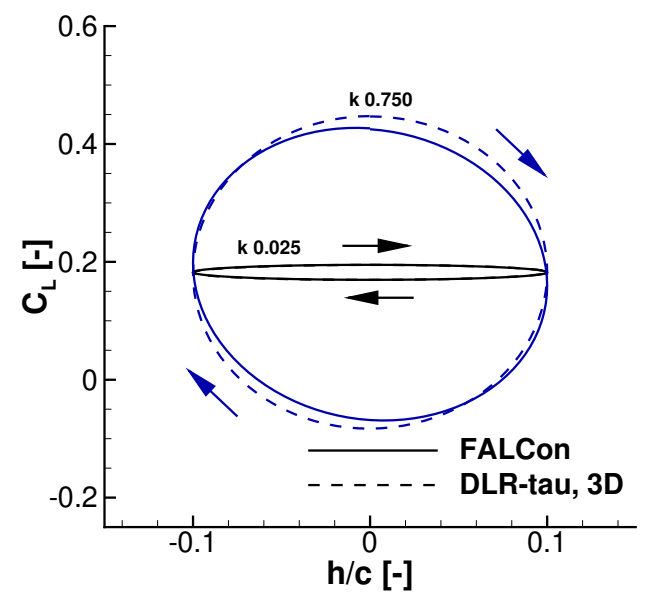

(a) $M=0.3$

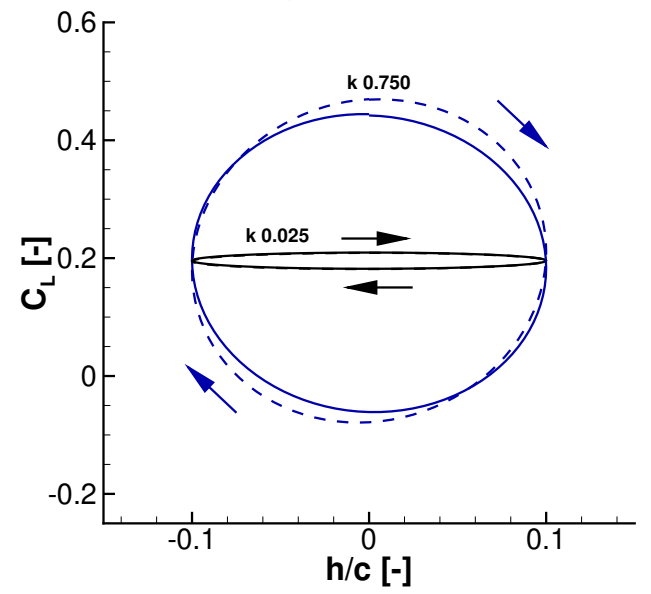

(c) $M=0.5$

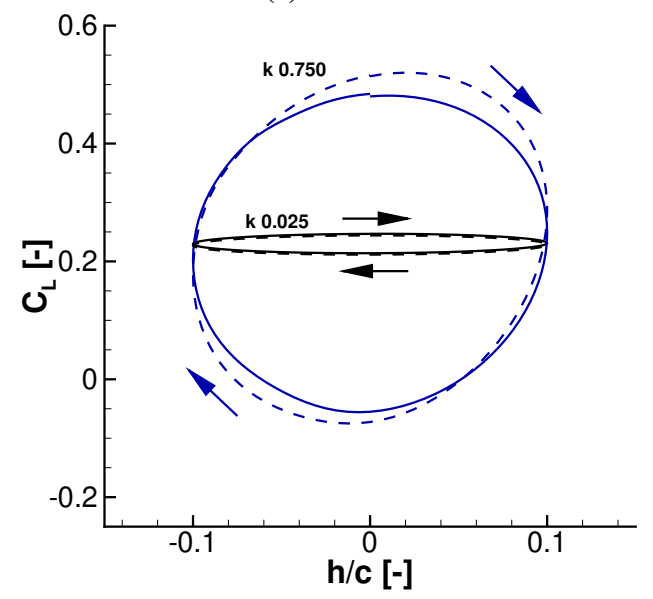

(e) $M=0.7$

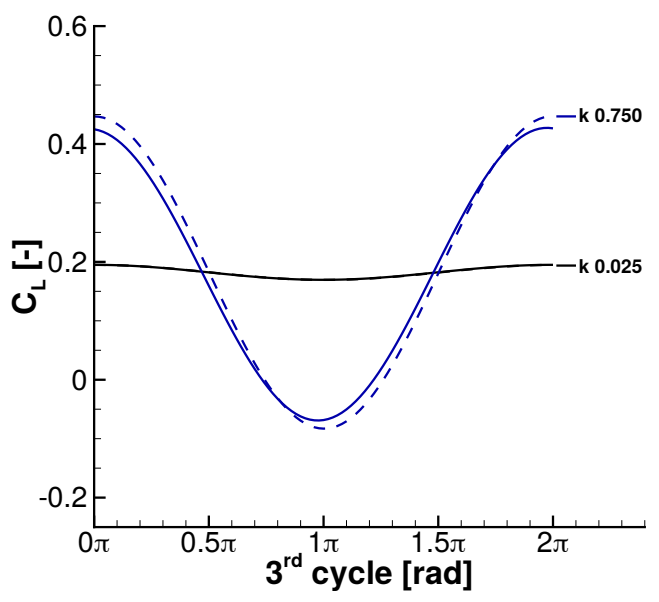

(b) $M=0.3$

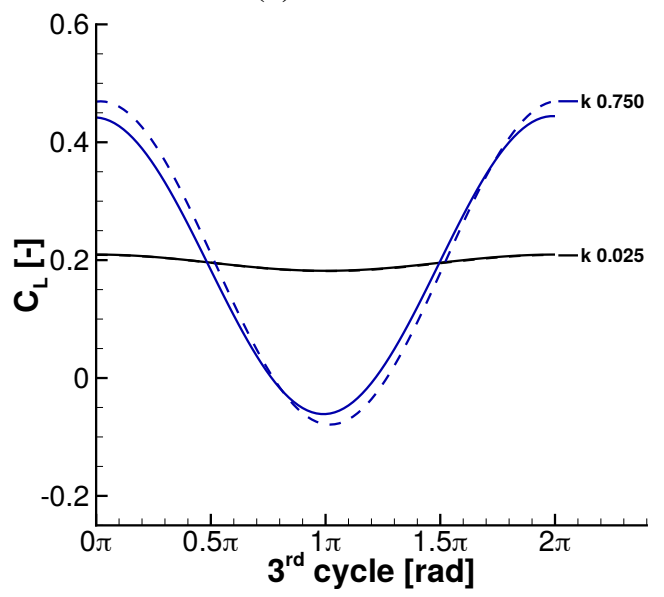

(d) $M=0.5$

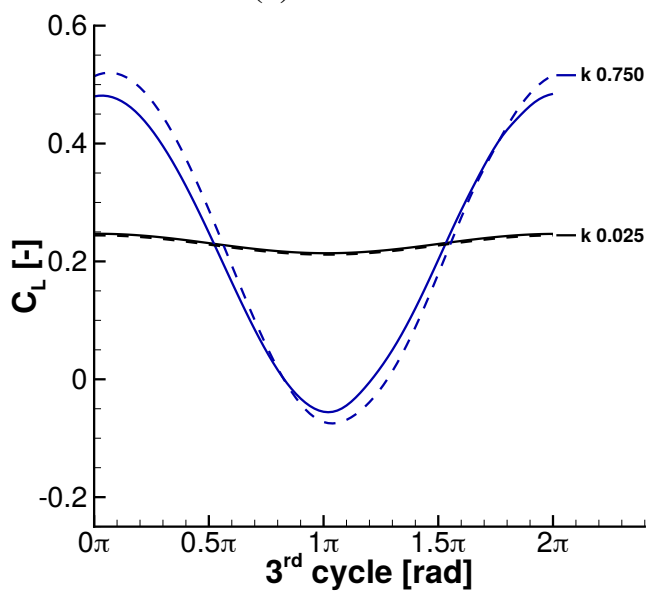

(f) $M=0.7$

Fig. 12 Test case B: lift coefficient hysteresis loops for forced plunge motion. In the legend, "DLR-tau, 3D" denotes reference data 


\begin{tabular}{ccccc}
\hline \hline & \multicolumn{2}{c}{ Pitch } & \multicolumn{2}{c}{ Plunge } \\
$k$ & 0.025 & 0.750 & 0.025 & 0.750 \\
\hline$\epsilon$ & 1.8 & 8.0 & 1.1 & 11.5 \\
\hline \hline
\end{tabular}

Table 2 Test case B: percent reconstruction error for forced motion in pitch and plunge at $M=0.7$

of attack provided by the unsteady coupling algorithm.

For further discussion, Figures 13 and 14 depict the instantaneous pressure coefficient for the pitch and plunge cases, respectively, at $M=0.7$. Figures 15 and 16 are the counterpart for the skin friction coefficient. To comprehend these articulated figures, rows correspond to time instants of a cycle and columns to three locations (25, 50 and $80 \%)$ along the wing span. The time snapshots capture the motion variable (either $\alpha$ or $\xi$ ) at four instants uniformly distributed within an oscillatory cycle: at the maximum value, the mean value during downstroke, the minimum value, and the mean value during upstroke. As an example, the fourth row of Figure 13 illustrates the instantaneous $C_{p}$ at stations located at 25, 50 and $80 \%$ of the wing span during the upstroke, for $\alpha=0^{\circ}$. The reconstruction of the instantaneous flow features is done well generally. It is not unexpected that the larger discrepancies in prediction are at the wing tip for $M=0.7$. These are due to the presence of a strong wing tip vortex forming around a straight wing, which is not a common design feature for application to high speed flows. A better agreement is therefore expected for an airliner wing which has a moderate sweep angle, reducing the strength of the tip vortex at high speed. Comparing pitch and plunge, differences seen in $C_{L}$ are attributed to deviations in the high speed region of the flow interacting with the wing tip vortex. As the reduced frequency of motion increases, FALCon predictions deviate on the suction peak of the pressure coefficient. We believe this to be attributed to the estimation of the induced angle of attack. A possible confirmation is found analysing the excellent aerofoil results in Section IV.A. where the induced angle of attack vanishes due to the 2D nature of that problem.

\section{Notes on Convergence}

The L2 norm of the residual of the hybrid aerodynamic solver FALCon is shown in Figure 17. An exemplary behaviour is for the forced motion in pitch at $M=0.7$ and $k=0.025$. The convergence behaviour is shown for the first six time steps of the motion, when the flow has yet to achieve periodicity. Convergence to the prescribed tolerance, $\varepsilon=10^{-6}$, occurs in just over 50 pseudo iterations, and the convergence rate seems unaffected as time progresses. A preliminary study was carried out and it was found that an optimal relaxation factor is $v=5 \cdot 10^{-2}$, in order to provide a good balance between convergence rate and number of iterations to convergence.

\section{Notes on Computational Cost}

The 3D URANS analysis required about 1,500 CPU hours. For FALCon, Part I of the unsteady coupling algorithm spawned nine 2D URANS analyses for angles of attack between -6 and $2^{\circ}$ in order to update the viscous database at 


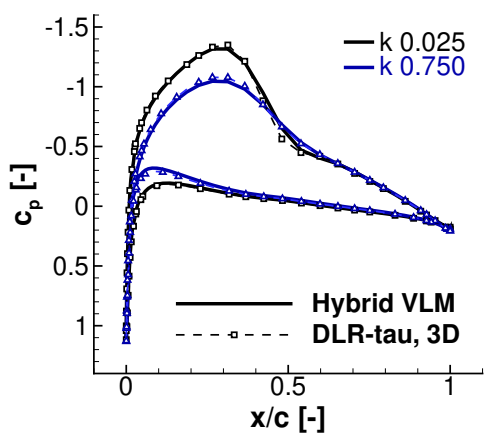

(a) $\alpha=2.51^{\circ}$ at $25 \%$ wing span

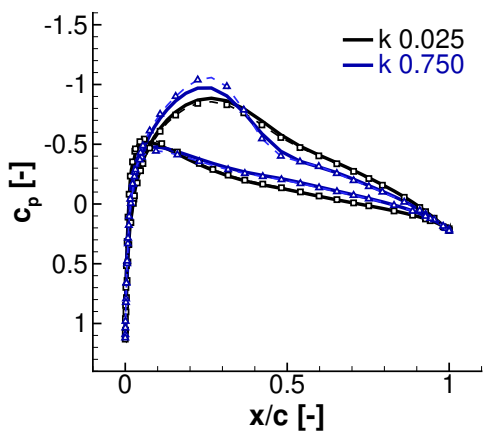

(d) $\alpha=0^{\circ}$ (down) at $25 \%$ wing span

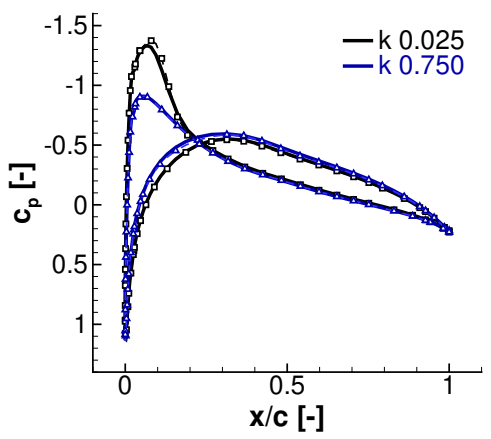

(g) $\alpha=-2.51^{\circ}$ at $25 \%$ wing span

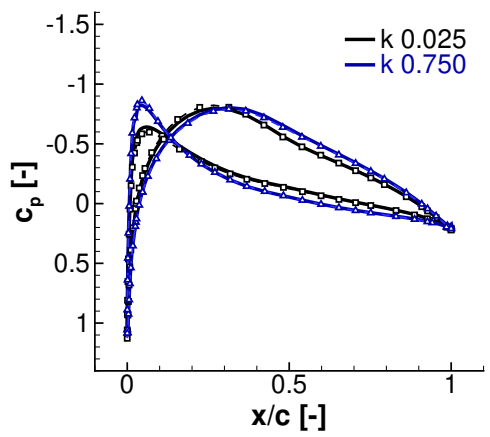

(j) $\alpha=0^{\circ}$ (up) at $25 \%$ wing span

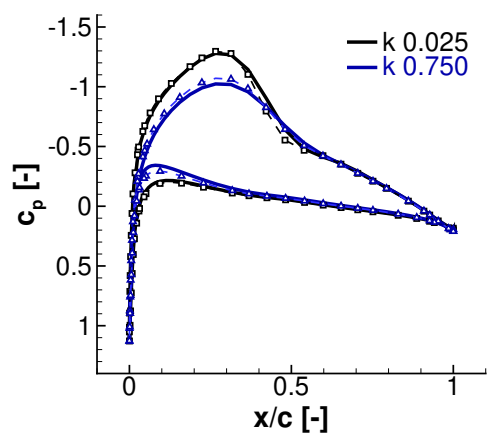

(b) $\alpha=2.51^{\circ}$ at $50 \%$ wing span

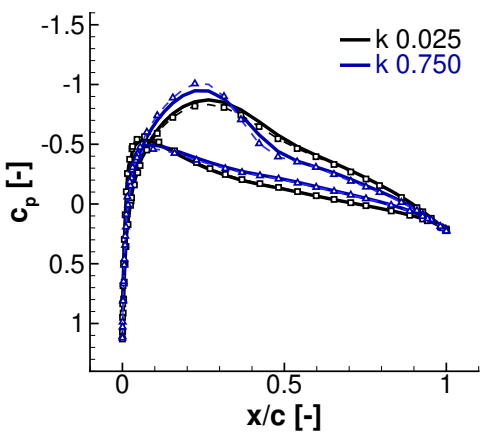

(e) $\alpha=0^{\circ}$ (down) at $50 \%$ wing span

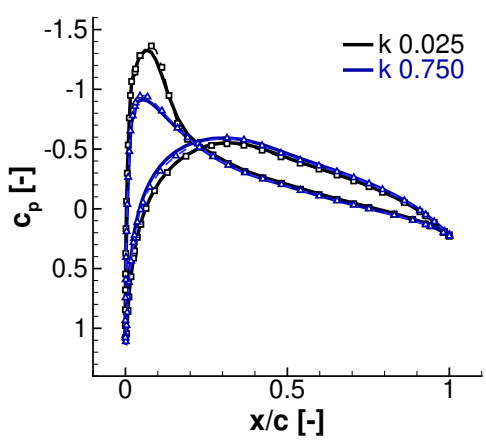

(h) $\alpha=-2.51^{\circ}$ at $50 \%$ wing span

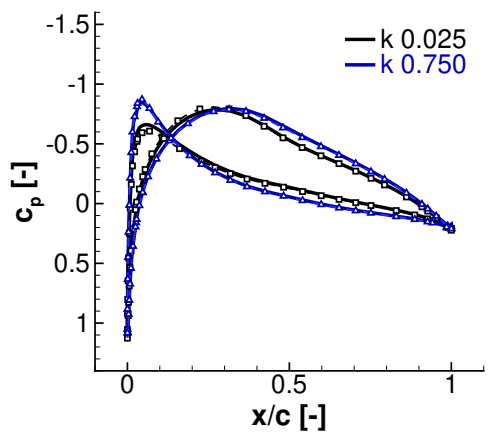

(k) $\alpha=0^{\circ}$ (up) at $50 \%$ wing span

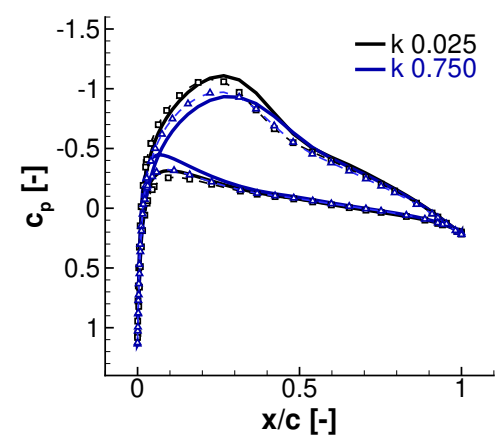

(c) $\alpha=2.51^{\circ}$ at $80 \%$ wing span

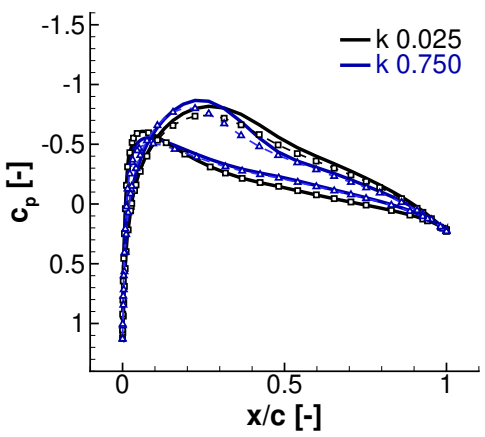

(f) $\alpha=0^{\circ}$ (down) at $80 \%$ wing span

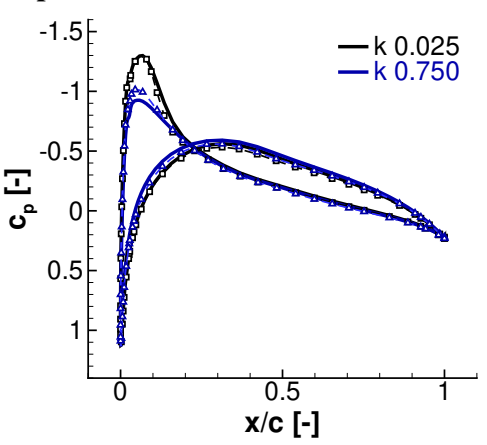

(i) $\alpha=-2.51^{\circ}$ at $80 \%$ wing span

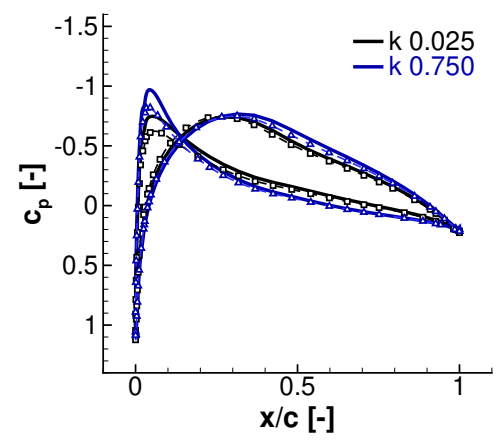

(l) $\alpha=0^{\circ}$ (up) at $80 \%$ wing span

Fig. 13 Test case B: instantaneous pressure coefficient for forced pitch motion at $M=0.7$. Rows depict time instants ("up" for upstroke and "down" for downstroke) and columns represent spanwise stations. In the legend, "DLR-tau, 3D" denotes reference data 

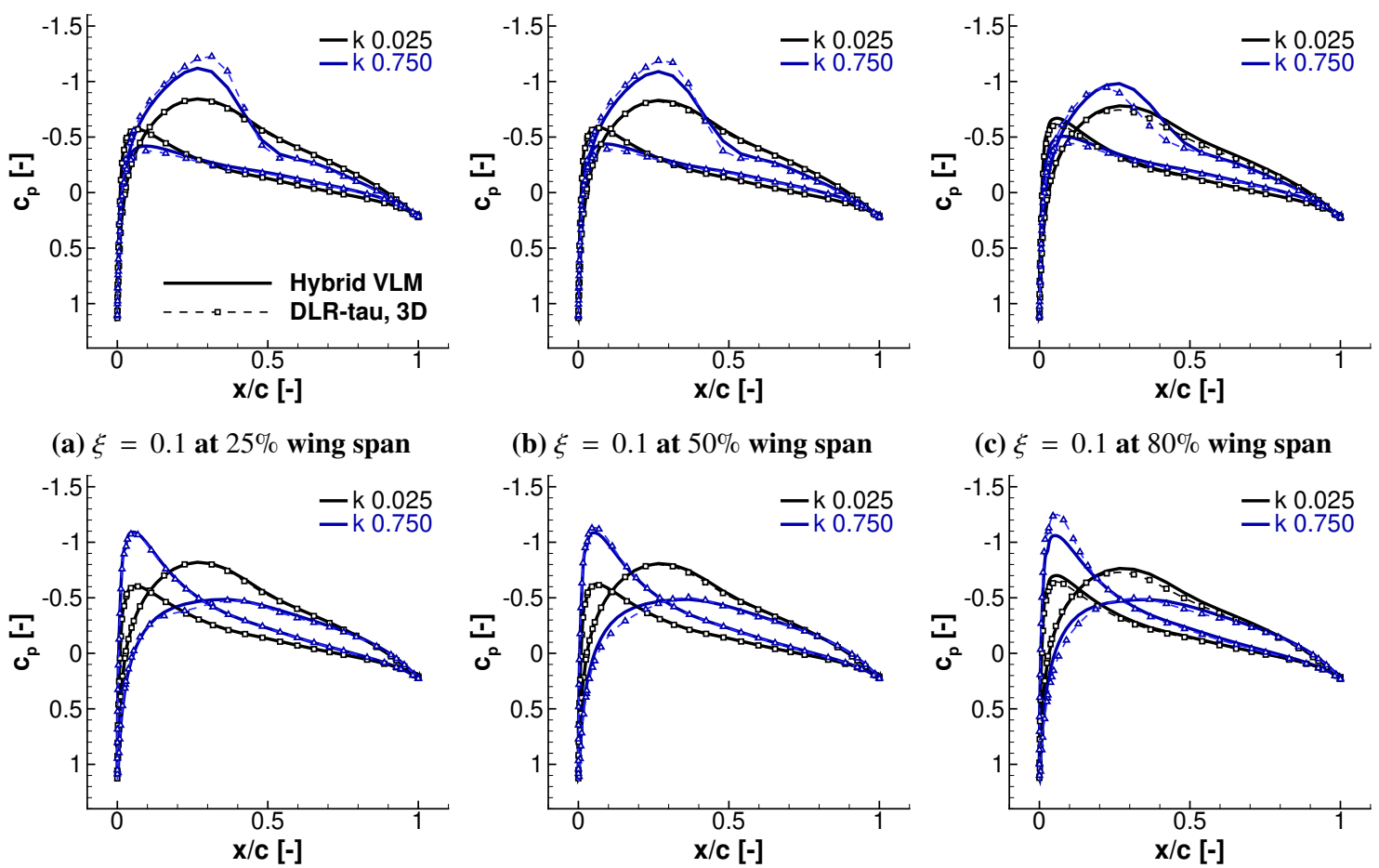

(c) $\xi=0.1$ at $80 \%$ wing span

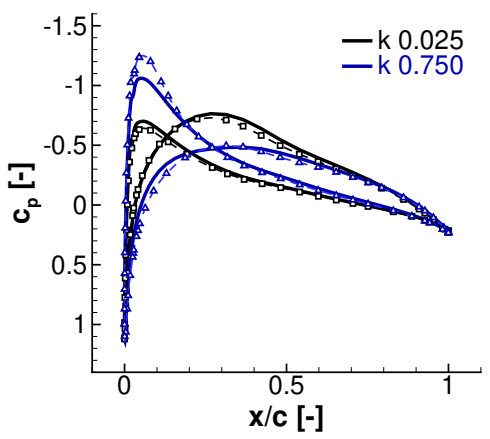

(d) $\xi=0$ (down) at $25 \%$ wing span

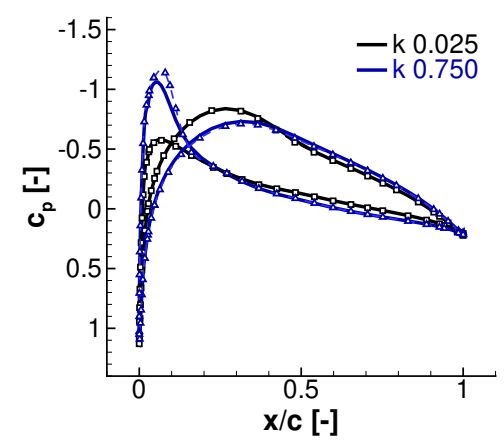

(e) $\xi=0$ at $50 \%$ wing span

(f) $\xi=0$ (down) at $80 \%$ wing span
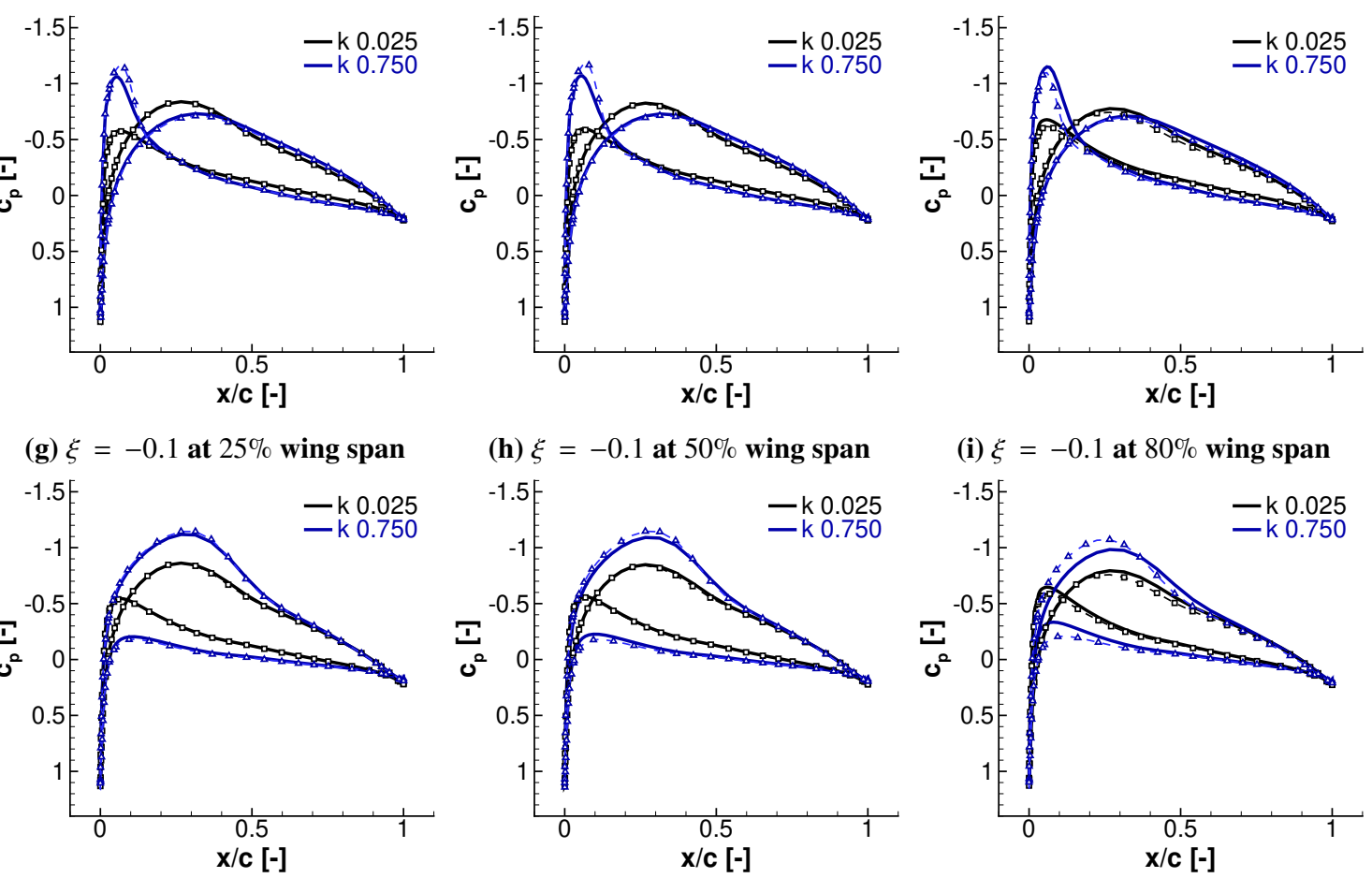

(h) $\xi=-0.1$ at $50 \%$ wing span
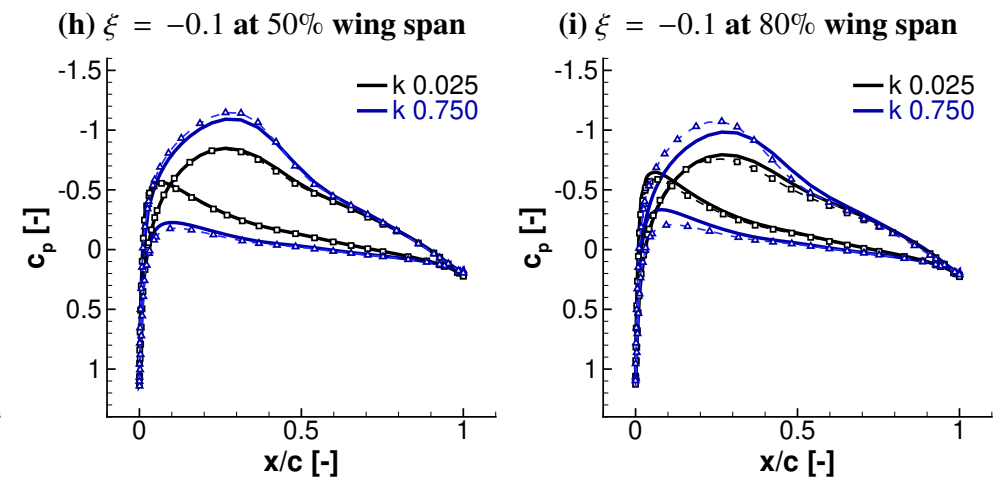

(j) $\xi=0$ (up) at $25 \%$ wing span

(k) $\xi=0$ (up) at $50 \%$ wing span

(l) $\xi=0$ (up) at $80 \%$ wing span

Fig. 14 Test case B: instantaneous pressure coefficient for forced plunge motion at $M=0.7$. Rows depict time instants ("up" for upstroke and "down" for downstroke) and columns represent spanwise stations. In the legend, "DLR-tau, 3D" denotes reference data 

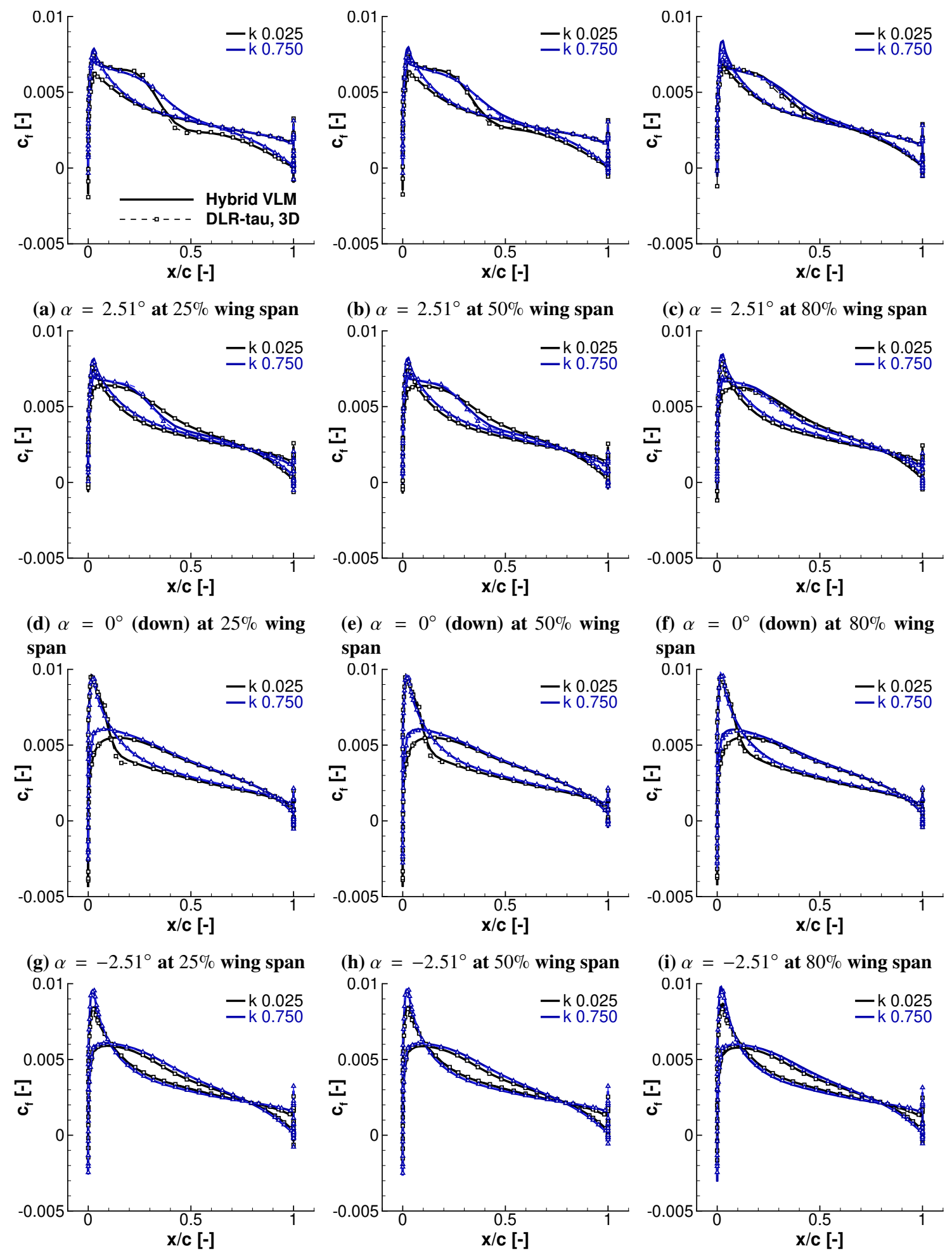

(j) $\alpha=0^{\circ}$ (up) at $25 \%$ wing span

(k) $\alpha=0^{\circ}$ (up) at $50 \%$ wing span

(l) $\alpha=0^{\circ}$ (up) at $80 \%$ wing span

Fig. 15 Test case B: instantaneous skin friction coefficient for forced pitch motion at $M=0.7$. Rows depict time instants ("up" for upstroke and "down" for downstroke) and columns represent spanwise stations. In the legend, "DLR-tau, 3D" denotes reference data 

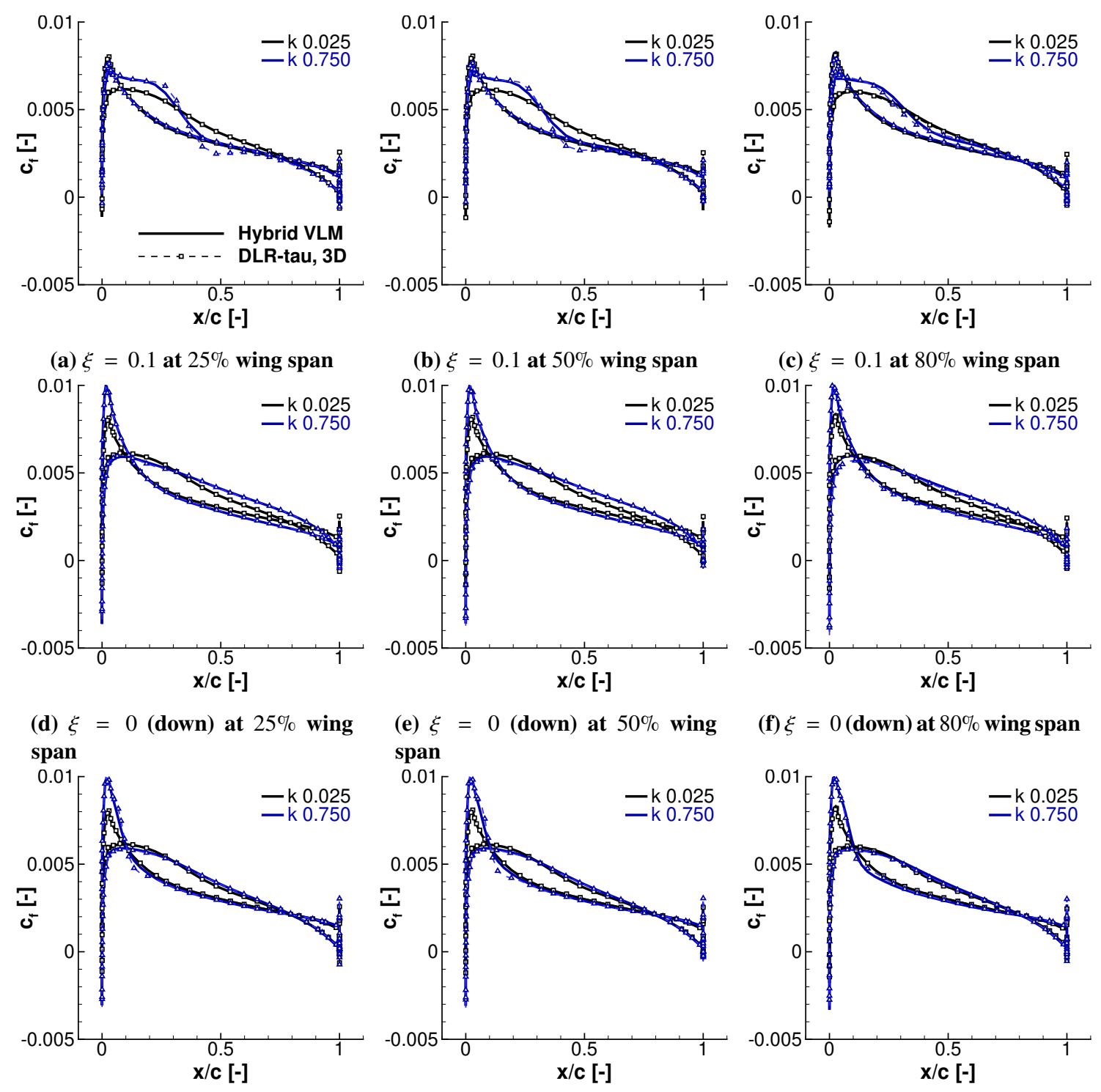

(f) $\xi=0($ down) at $80 \%$ wing span
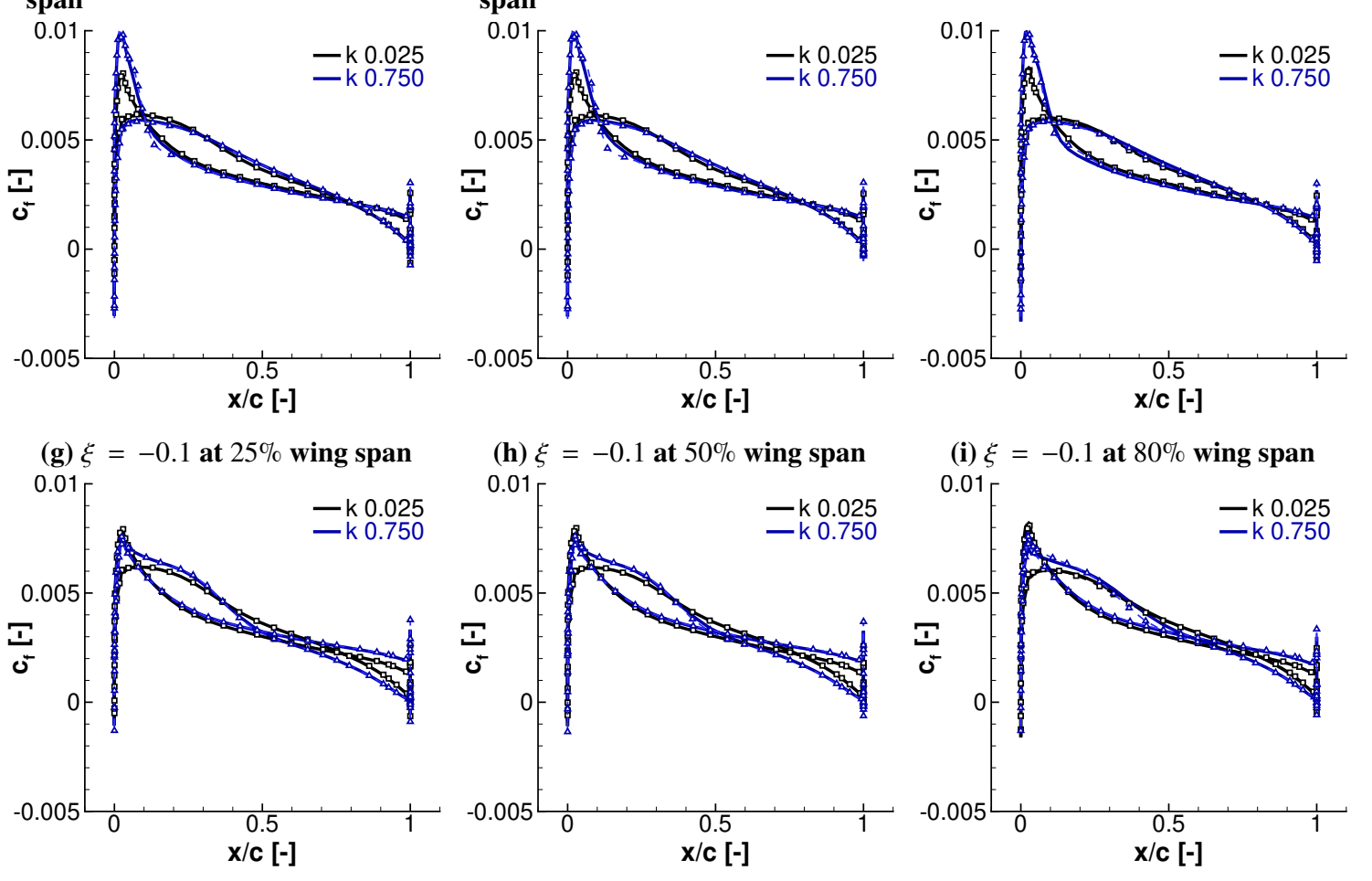

(j) $\xi=0$ (up) at $25 \%$ wing span

(k) $\xi=0$ (up) at $50 \%$ wing span

(l) $\xi=0$ (up) at $80 \%$ wing span

Fig. 16 Test case B: instantaneous skin friction coefficient for forced plunge motion at $M=0.7$. Rows depict time instants ("up" for upstroke and "down" for downstroke) and columns represent spanwise stations. In the legend, "DLR-tau, 3D" denotes reference data 


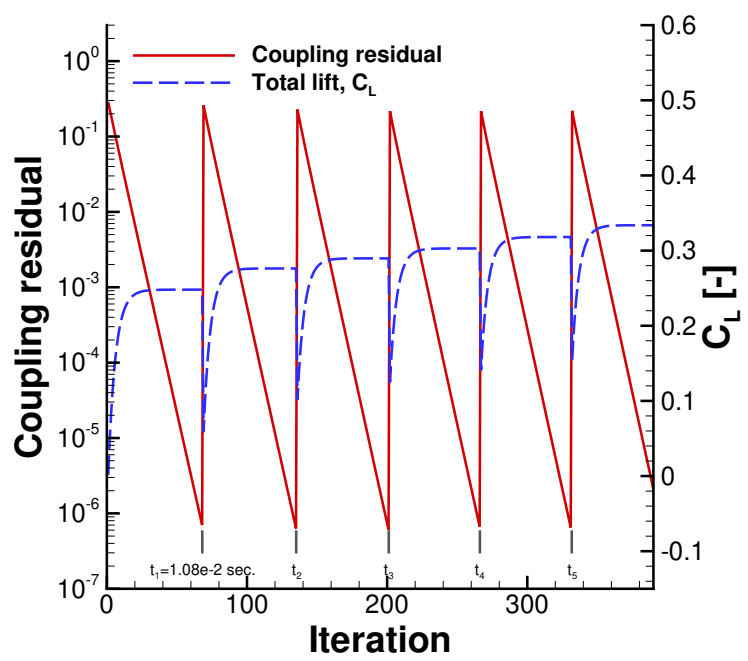

Fig. 17 Test case B: convergence of the hybrid aerodynamic solver during the first 6 time steps for forced pitch motion at $M=0.7$ and $k=0.025$

each time step. The 2D URANS analyses were run in parallel and required a total of 28 CPU hours. As both the UVLM and Part II of the unsteady coupling algorithm are inexpensive, it is conservative to indicate that FALCon required less than 30 CPU hours. Therefore, predictions obtained using the hybrid aerodynamic solver are, on average, a factor of 50 times faster than the results from time marching the 3D URANS equations. This significant speedup becomes a tangible asset of the proposed aerodynamic model when balanced against the accuracy of predictions for a number of valuable test cases.

\section{Forced harmonic motion in pitch: finite-span wing in transonic flow}

The final demonstration test case features a pitching wing exposed to a $M=0.8$ air flow. The wing geometry and the pitch motion kinematics are the same as those described in Section IV.B. The complex nature of the resulting flow provides the opportunity to assess the predictive performance of the hybrid aerodynamic solver on a problem well beyond its underlying assumptions. In this regard, consider Figure 18 that depicts the temporal evolution of areas with flow separation.

The flow physics is characterised by the appearance and disappearance of a shock wave which locks-in with the periodic motion and causes the flow to separate. The shock moves periodically and extends uniformly in the spanwise direction until it interacts with the vortex emanating at the wing tip. These interactions and the complex dynamics resulting from the forced motion go beyond the assumptions (most notably, of uniform flow) of the hybrid aerodynamic model. Therefore, limitations of the proposed aerodynamic model will start to appear.

For completeness, FALCon predictions are summarised in Figures 19 and 20 for the pressure coefficient and skin friction coefficient, respectively. One observes that the flow reconstruction at lower frequency outperforms the one at higher frequency. This is in line with Figure 18 conveying a stronger flow three-dimensionality for $k=0.750$. For 
$k=0.025$, flow predictions are generally good. Some discrepancies between the two sources of data are visible on the outboard section during the upstroke and downstroke. For $k=0.750$, one finds that FALCon predictions become less reliable at the higher angles of attack of the oscillatory cycle (first and second rows). Fortunately, this unfavourable situation carries a more academic interest than a practical relevance because transonic wings are designed with a moderate sweep angle to relieve the problematic associated with the system of shocks and vortices.

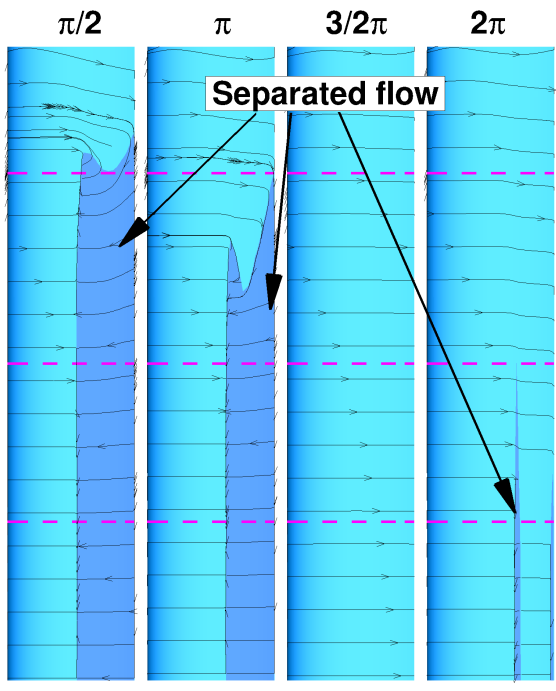

(a) $k=0.025$
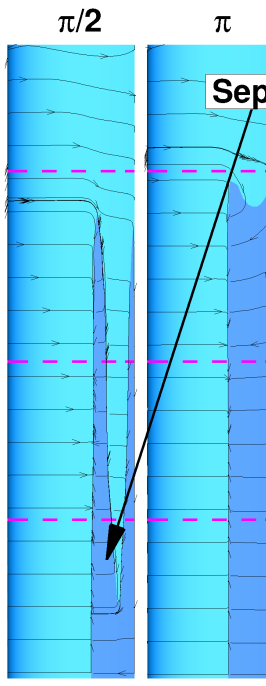

(b) $k=0.750$

Fig. 18 Test case C: surface contour of skin friction coefficient from 3D URANS analysis revealing appearance and disappearance of flow separation locked-in with forced pitch motion at $M=0.8$ 


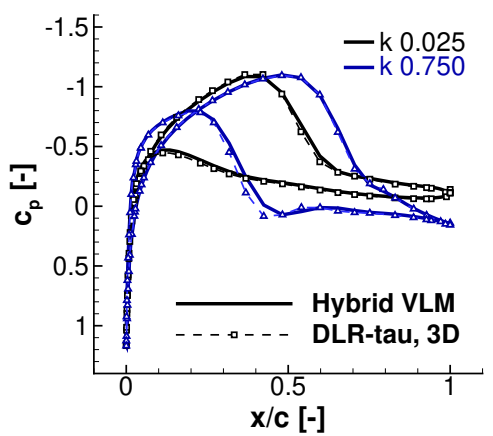

(a) $\alpha=2.51^{\circ}$ at $25 \%$ wing span

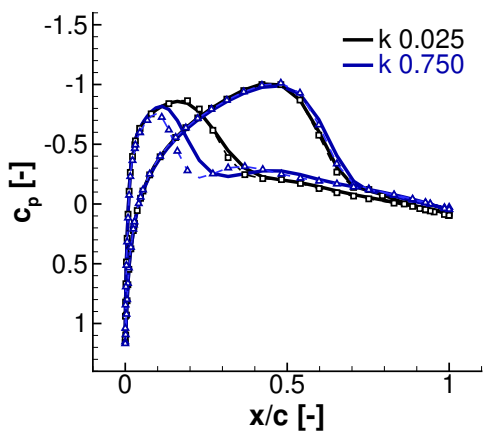

(d) $\alpha=0^{\circ}$ (down) at $25 \%$ wing span

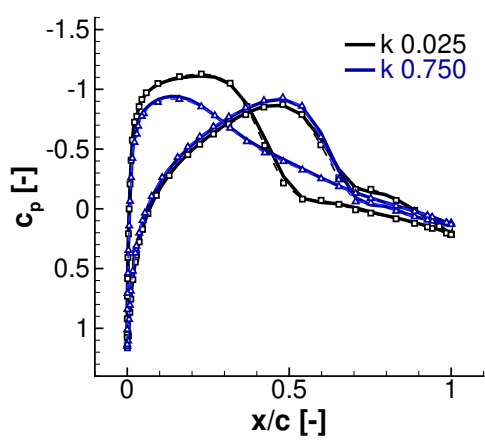

(g) $\alpha=-2.51^{\circ}$ at $25 \%$ wing span

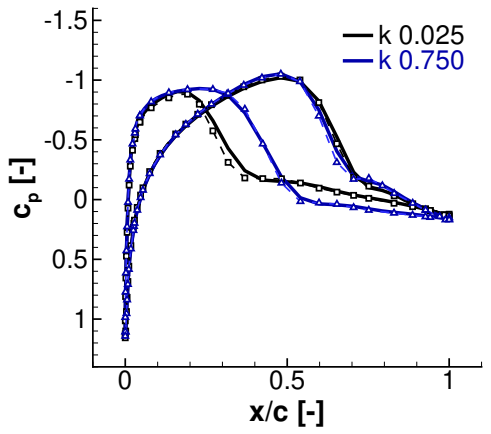

(j) $\alpha=0^{\circ}$ (up) at $25 \%$ wing span

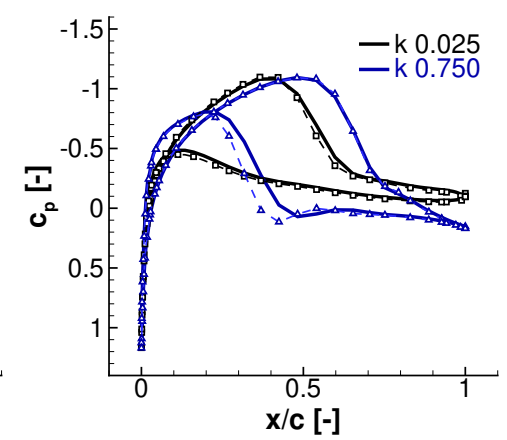

(b) $\alpha=2.51^{\circ}$ at $50 \%$ wing span

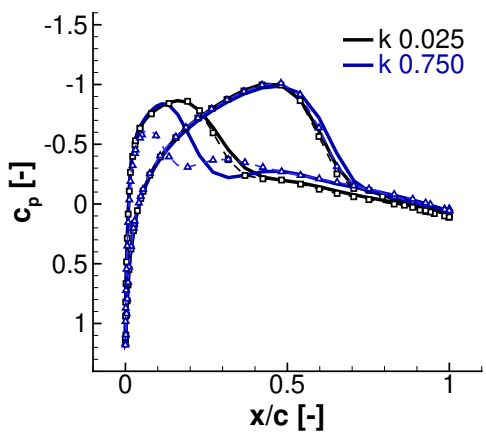

(e) $\alpha=0^{\circ}$ (down) at $50 \%$ wing span

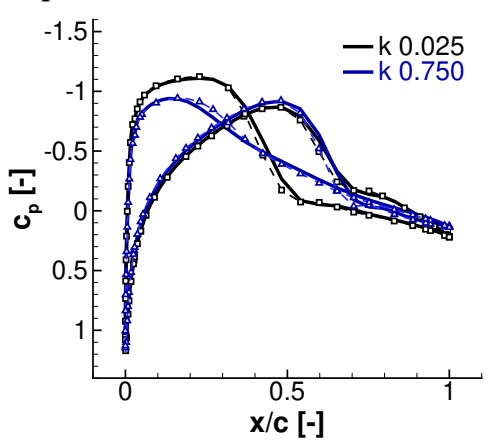

(h) $\alpha=-2.51^{\circ}$ at $50 \%$ wing span

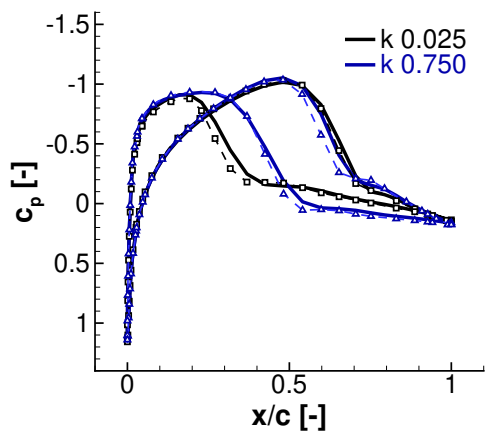

(k) $\alpha=0^{\circ}$ (up) at $50 \%$ wing span

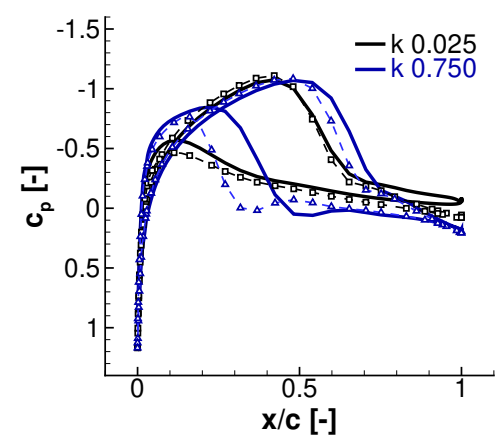

(c) $\alpha=2.51^{\circ}$ at $80 \%$ wing span

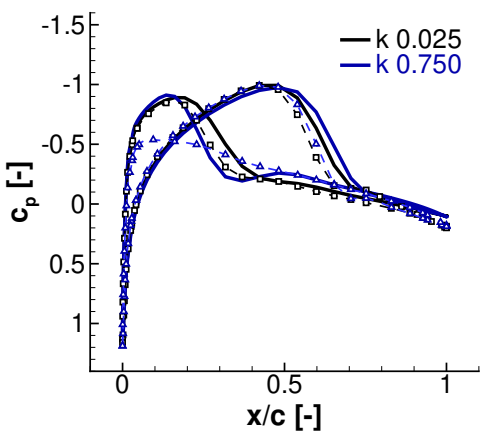

(f) $\alpha=0^{\circ}$ (down) at $80 \%$ wing span

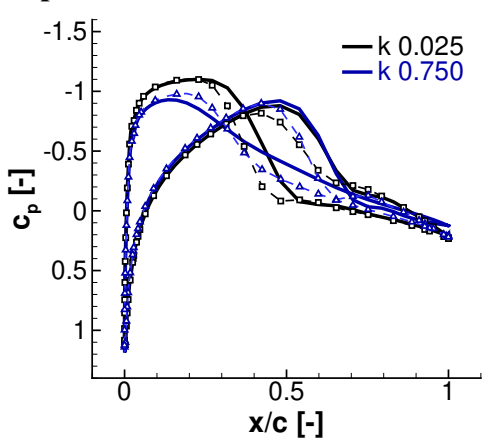

(i) $\alpha=-2.51^{\circ}$ at $80 \%$ wing span

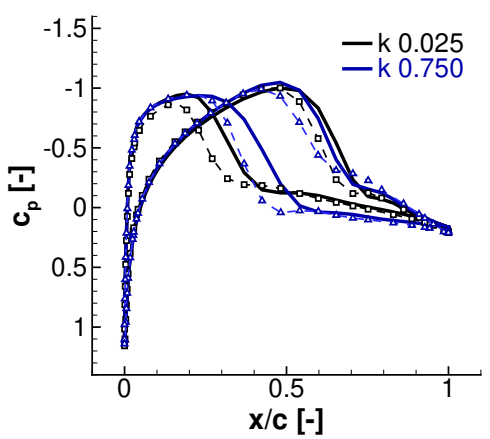

(l) $\alpha=0^{\circ}$ (up) at $80 \%$ wing span

Fig. 19 Test case $\mathrm{C}$ : instantaneous pressure coefficient for forced pitch motion at $M=0.8$. Rows depict time instants ("up" for upstroke and "down" for downstroke) and columns represent spanwise stations. In the legend, "DLR-tau, 3D" denotes reference data 

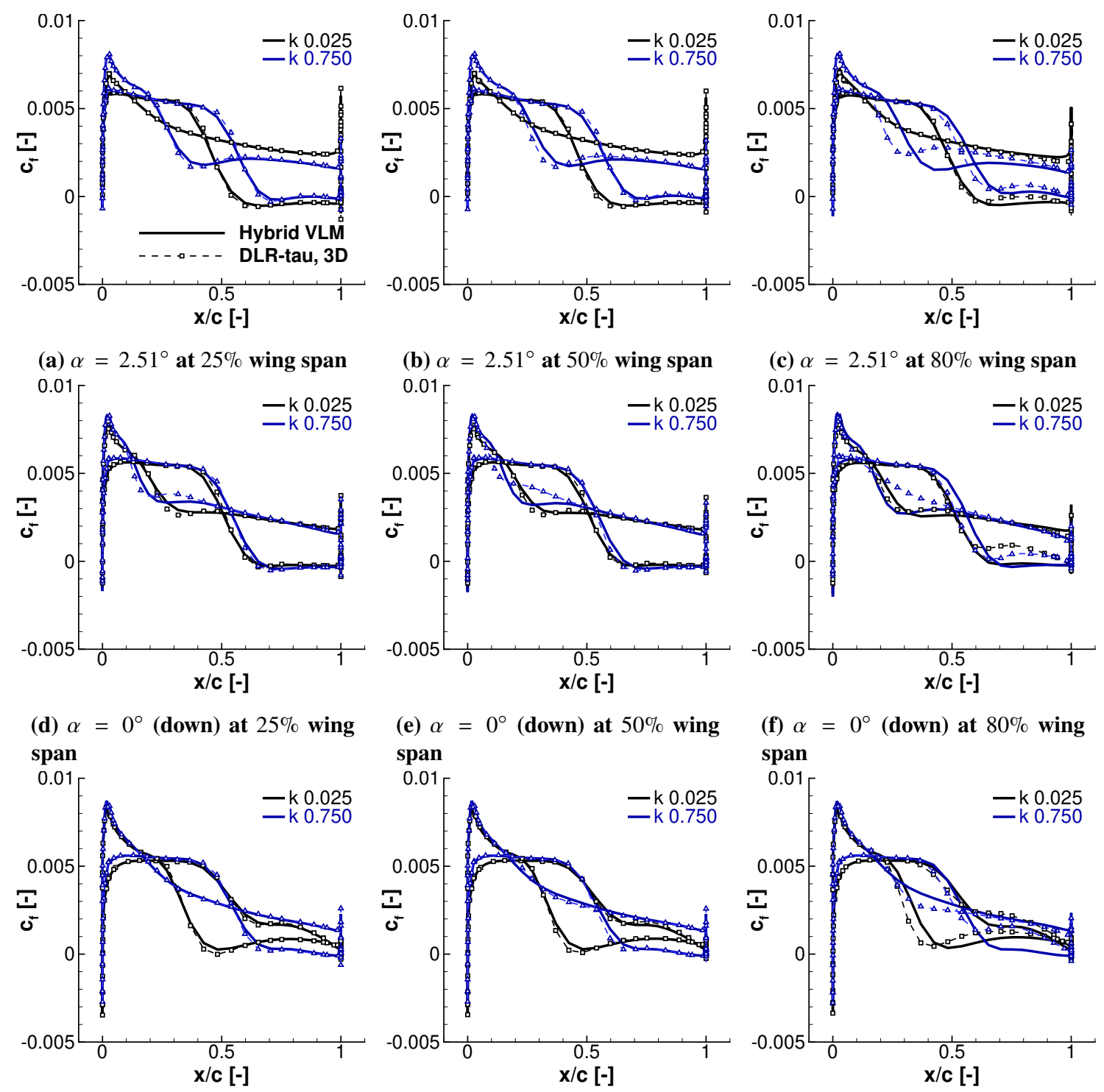

(f) $\alpha=0^{\circ}$ (down) at $80 \%$ wing
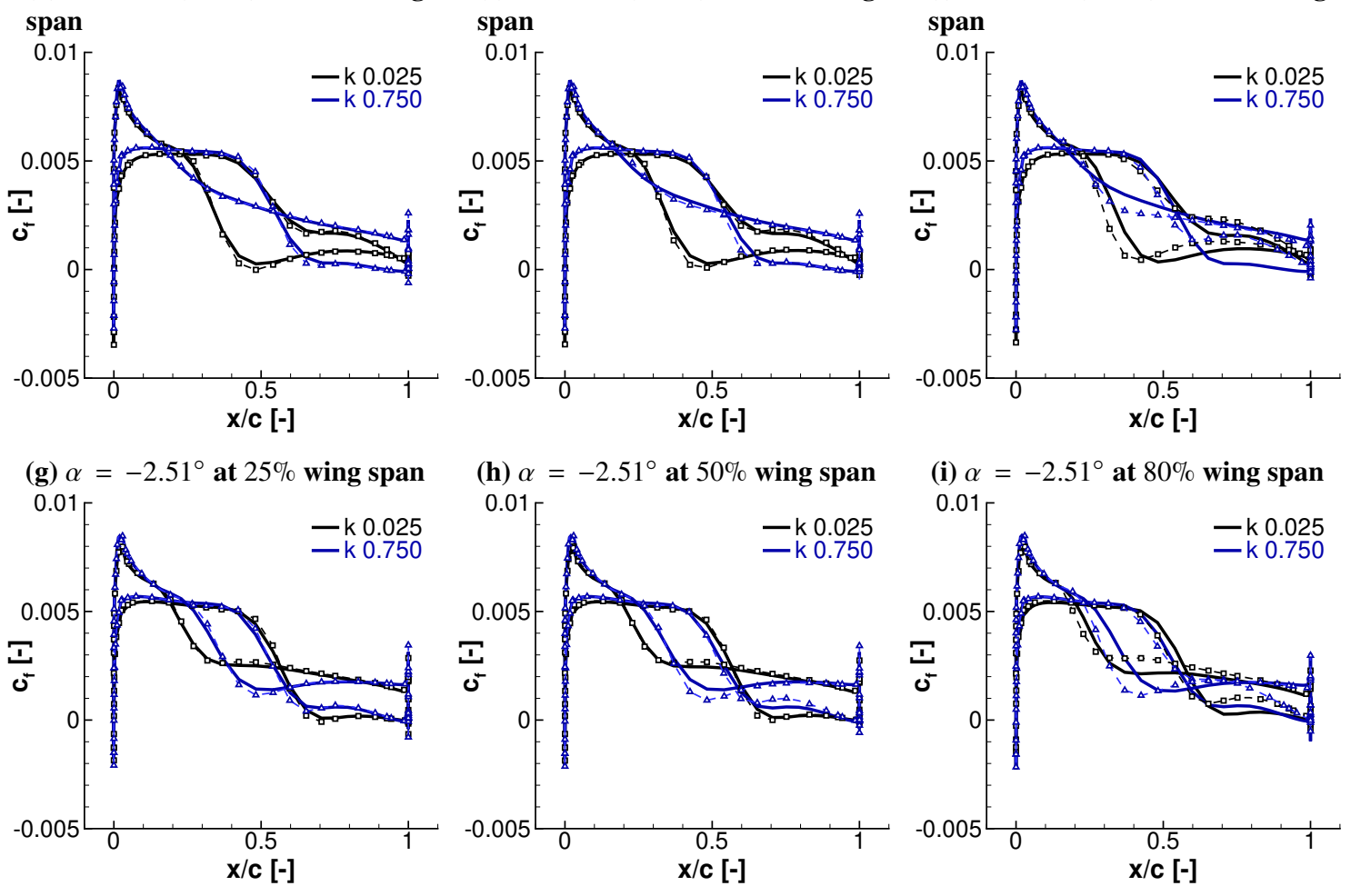

(j) $\alpha=0^{\circ}$ (up) at $25 \%$ wing span

(k) $\alpha=0^{\circ}$ (up) at $50 \%$ wing span

(l) $\alpha=0^{\circ}$ (up) at $80 \%$ wing span

Fig. 20 Test case $\mathbf{C}$ : instantaneous skin friction coefficient for forced pitch motion at $M=0.8$. Rows depict time instants ("up" for upstroke and "down" for downstroke) and columns represent spanwise stations. In the legend, "DLR-tau, 3D" denotes reference data 


\section{Conclusion}

The paper addressed the problem of enhancing the predictive capability of a potential flow-based aerodynamic model with viscous sectional information. Whereas the existing knowledge and practice is well-established for steady-state problems, the same cannot be stated for unsteady flows. The contribution of this research work is the extension of the $\alpha$-based coupling algorithm to unsteady problems. The unsteady coupling algorithm is formulated in such a way to permit any type of wing motion, outperforming prior attempts limited by the motion kinematics and restricted to low frequencies of motion. The algorithm consists of two main operation blocks: Part I which concerns data generation from two specialised and separate aerodynamic models that are synchronously marched forward in time, and Part II which executes data fusion to produce more consistent and accurate information than that provided by any individual aerodynamic source. The aerodynamic models used herein are an in-house unsteady vortex lattice method and an infinite-swept wing Navier-Stokes solver. We have named the resulting computational tool FALCon, as an acronym for Fast Aircraft Load Calculations. A preliminary study was carried out to validate the two aerodynamic models and the unsteady coupling algorithm. Then, the predictive performance capability of FALCon was demonstrated on three test cases of increasing complexity. Test case A featured an unsteady, two-dimensional flow problem which was superseded by a family of three-dimensional flow problems in Test case B. Predictions matched well reference data for all Mach numbers tested and for both low and high reduced frequencies of motion (up to $k=0.75$ ). Test case $\mathrm{C}$ superseded previous cases and featured flows with a strong three-dimensional character caused by a shock wave interacting with areas of flow separation and with the wing tip vortex, driven by the prescribed motion of the wing. Test case $\mathrm{C}$ was chosen to exercise FALCon on problems where the underlying assumption (i.e. uniform or slowly changing flow along the span) is not met. An acceptable agreement was found, balanced by a speed-up of a factor of about 50 compared to solving in time the three-dimensional Reynolds-averaged Navier-Stokes equations. FALCon reproduced important physical features, such as the influence of compressibility and flow unsteadiness on the aerodynamic stiffness and damping, prerequisites in any aeroelastic analysis. Finally, it is worth noting that alternative aerodynamic models may be considered as a replacement for the specific inviscid and viscous models here discussed.

\section{Acknowledgements}

Da Ronch acknowledges the financial support from the Engineering and Physical Sciences Research Council (grant number: EP/P006795/1), the Royal Academy of Engineering (grant number: ISS1415/7/44) and Airbus Operations SAS. The authors acknowledge the use of the IRIDIS High-Performance Computing Facility, and associated support services at the University of Southampton, in the completion of this work.

Data supporting this study will be openly available from the University of Southampton repository after publication. 


\section{References}

[1] Badcock, K. J., Timme, S., Marques, S., Khodaparast, H., Prandina, M., Mottershead, J. E., Swift, A., Da Ronch, A., and Woodgate, M. A., "Transonic aeroelastic simulation for instability searches and uncertainty analysis," Progress in Aerospace Sciences, Vol. 47, No. 5, 2011, pp. 392-423. doi:10.1016/j.paerosci.2011.05.002.

[2] Tani, I., A simple method of calculating the induced velocity of a monoplane wing, Aeronautical Research Institute, Tokyo Imperial University, 1934.

[3] Multhopp, H., and Schwabe, M., Die Berechnung der Auftriebsverteilung von Tragflügeln, 1938.

[4] Sivells, J. C., and Neely, R. H., "Method for calculating wing characteristics by lifting-line theory using nonlinear section lift data,” Tech. rep., DTIC Document, 1947.

[5] Sivells, J. C., and Westrick, G. C., "Method for Caluculating Lift Distributions for Unswept Wings with Flaps or Ailerons by use of Nonlinear Section Lift Data," 1951.

[6] Tseng, J.-B., and Lan, C. E., "Calculation of aerodynamic characteristics of airplane configurations at high angles of attack," 1988.

[7] Dam, C. V., "The aerodynamic design of multi-element high-lift systems for transport airplanes," Progress in Aerospace Sciences, Vol. 38, No. 2, 2002, pp. 101 - 144. doi:10.1016/S0376-0421(02)00002-7.

[8] Van Dam, C., Vander Kam, J., and Paris, J., "Design-oriented high-lift methodology for general aviation and civil transport aircraft," Journal of aircraft, Vol. 38, No. 6, 2001, pp. 1076-1084.

[9] Gallay, S., and Laurendeau, E., "Nonlinear Generalized Lifting-Line Coupling Algorithms for Pre/Poststall Flows," AIAA Journal, Vol. 53, No. 7, 2015, pp. 1784-1792. doi:10.2514/1.J053530.

[10] Gallay, S., and Laurendeau, E., "Preliminary-Design Aerodynamic Model for Complex Configurations Using Lifting-Line Coupling Algorithm,” Journal of Aircraft, Vol. 53, No. 4, 2016, pp. 1145-1159.

[11] Parenteau, M., Plante, F., Laurendeau, E., and Costes, M., "Unsteady Coupling Algorithm for Lifting-Line Methods," 55th AIAA Aerospace Sciences Meeting, 2017, p. 0951.

[12] Da Ronch, A., Ghoreyshi, M., and Badcock, K., "On the generation of flight dynamics aerodynamic tables by computational fluid dynamics," Progress in Aerospace Sciences, Vol. 47, No. 8, 2011, pp. 597-620. doi:10.1016/j.paerosci.2011.09.001.

[13] Da Ronch, A., Vallespin, D., Ghoreyshi, M., and Badcock, K., "Evaluation of dynamic derivatives using computational fluid dynamics,” AIAA Journal, Vol. 50, No. 2, 2012, pp. 470-484. doi:10.2514/1.J051304.

[14] Da Ronch, A., Ghoreyshi, M., Badcock, K. J., Görtz, S., Widhalm, M., Dwight, R. P., and Campobasso, M. S., "Linear frequency domain and harmonic balance predictions of dynamic derivatives,” Journal of Aircraft, Vol. 50, No. 3, 2013, pp. 694-707. doi:10.2514/1.C031674. 
[15] Ghoreyshi, M., Badcock, K. J., Da Ronch, A., Marques, S., Swift, A., and Ames, N., "Framework for establishing limits of tabular aerodynamic models for flight dynamics analysis," Journal of Aircraft, Vol. 48, No. 1, 2011, pp. 42-55. doi:10.2514/1.C001003.

[16] Eldredge, J. D., Colonius, T., and Williams, D., "EnKF-based Dynamic Estimation of Separated Flows with a Low-Order Vortex Model,” AIAA SciTech Forum, 2018. AIAA Paper 2018-0811.

[17] Le Provost, M., Hou, W., and Eldredge, J. D., "Deep learning and data assimilation approaches to sensor reduction in estimation of disturbed separated flows," AIAA SciTech Forum, 2020. AIAA Paper 2020-0799.

[18] Clark Di Leoni, P., Mazzino, A., and Biferale, L., "Synchronization to Big Data: Nudging the Navier-Stokes Equations for Data Assimilation of Turbulent Flows,” Physical Review X, Vol. 10, No. 1, 2020. doi:10.1103/physrevx.10.011023.

[19] Bagheri, A. K., and Da Ronch, A., "Adjoint-Based Surrogate Modelling of Spalart-Allmaras Turbulence Model Using Gradient Enhanced Kriging,” AIAA Aviation Forum, 2020. AIAA Paper 2020-2991.

[20] Katz, J., and Plotkin, A., Low-speed aerodynamics, Vol. 13, Cambridge University Press, 2001.

[21] Murua, J., Palacios, R., and Graham, J. M. R., "Applications of the unsteady vortex-lattice method in aircraft aeroelasticity and flight dynamics," Progress in Aerospace Sciences, Vol. 55, No. Supplement C, 2012, pp. 46 - 72. doi:10.1016/j.paerosci.2012. 06.001

[22] Simpson, R. J. S., Palacios, R., and Murua, J., "Induced-Drag Calculations in the Unsteady Vortex Lattice Method," AIAA Journal, Vol. 51, No. 7, 2013, pp. 1775-1779. doi:10.2514/1.J052136.

[23] Franciolini, M., Da Ronch, A., Drofelnik, J., Raveh, D., and Crivellini, A., "Efficient infinite-swept wing solver for steady and unsteady compressible flows," Aerospace Science and Technology, Vol. 72, 2018, pp. 217 - 229. doi:10.1016/j.ast.2017.10.034.

[24] Drofelnik, J., Da Ronch, A., Franciolini, M., and Crivellini, A., "Fast identification of transonic buffet envelope using computational fluid dynamics," Aircraft Engineering and Aerospace Technology, Vol. 91, No. 2, 2019, pp. 309-316. doi: 10.1108/AEAT-01-2018-0057.

[25] Yang, G., Da Ronch, A., Kharlamov, D., and Drofelnik, J., "Wing twist optimisation using aerodynamic solvers of different fidelity," 31st Congress of the International Council of the Aeronautical Sciences (ICAS 2018), 2018. URL https://eprints.soton.ac.uk/423722/

[26] Jameson, A., Schmidt, W., and Turkel, E., "Numerical solutions of the Euler equations by finite volume methods using RungeKutta time-stepping schemes," 14th Fluid and Plasma Dynamics Conference, Fluid Dynamics and Co-located Conferences, Palo Alto, CA, 1981. doi:10.2514/6.1981-1259.

[27] Leishman, J., and Crouse, G., "State-space model for unsteady airfoil behavior and dynamic stall," Structures, Structural Dynamics, and Materials and Co-located Conferences, 1989. doi:10.2514/6.1989-1319, 0.

[28] Lambert, T., Abdul Razak, N., and Dimitriadis, G., "Vortex Lattice Simulations of Attached and Separated Flows around Flapping Wings," Aerospace, Vol. 4, No. 2, 2017. doi:10.3390/aerospace4020022, URL http://www .mdpi . com/2226-4310/4/2/22 
[29] Murua, J., "Flexible Aircraft Dynamics with a Geometrically-Nonlinear Description of the Unsteady Aerodynamics,” Ph.D. thesis, Imperial College London, United Kingdom, 2012.

[30] Theodorsen, T., and Mutchler, W., "General theory of aerodynamic instability and the mechanism of flutter," 1935.

[31] Spalart, P., and Allmaras, S., “A One-Equation Turbulence Model for Aerodynamic Flows,” AIAA, Vol. 439, 1992. doi: 10.2514/6.1992-439. 